\title{
SWITCHING AND STABILITY PROPERTIES OF CONEWISE LINEAR SYSTEMS*,**
}

\author{
Jinglai Shen $^{1}$, LANShan HAN ${ }^{2}$ AND Jong-Shi PANG ${ }^{2}$
}

\begin{abstract}
Being a unique phenomenon in hybrid systems, mode switch is of fundamental importance in dynamic and control analysis. In this paper, we focus on global long-time switching and stability properties of conewise linear systems (CLSs), which are a class of linear hybrid systems subject to state-triggered switchings recently introduced for modeling piecewise linear systems. By exploiting the conic subdivision structure, the "simple switching behavior" of the CLSs is proved. The infinitetime mode switching behavior of the CLSs is shown to be critically dependent on two attracting cones associated with each mode; fundamental properties of such cones are investigated. Verifiable necessary and sufficient conditions are derived for the CLSs with infinite mode switches. Switch-free CLSs are also characterized by exploring the polyhedral structure and the global dynamical properties. The equivalence of asymptotic and exponential stability of the CLSs is established via the uniform asymptotic stability of the CLSs that in turn is proved by the continuous solution dependence on initial conditions. Finally, necessary and sufficient stability conditions are obtained for switch-free CLSs.
\end{abstract}

Mathematics Subject Classification. 93B12, 93D05, 93D20.

Received September 19, 2008. Revised February 15, 2009.

Published online July 2nd, 2009.

\section{INTRODUCTION}

A conewise linear system (CLS) is a dynamical system consisting of a finite number of linear dynamical systems that are active on certain polyhedral cones which partition the entire Euclidean state space [11]. Each of these linear systems is called a mode of the CLS; transitions between modes occur along a state trajectory. Introduced in [11] for modeling Lipschitz piecewise linear systems, the CLSs form a special class of linear hybrid automata [24], where the vector fields are linear in states, the invariant sets are solid polyhedral cones, the guard

\footnotetext{
Keywords and phrases. Variable structure systems, Lyapunov and other classical stabilities, asymptotic stability.

* The research of J. Shen is partially supported by UMBC Summer Faculty Fellowship.

** The work of J.-S. Pang is based on research supported by the National Science Foundation under grant DMS-0508986.

1 Department of Mathematics and Statistics, University of Maryland Baltimore County, Baltimore, Maryland 21250, USA. shenj@umbc.edu

2 Department of Industrial and Enterprise Systems Engineering, University of Illinois at Urbana-Champaign, Urbana, Illinois 61801, USA. hanlsh@illinois.edu; jspang@illinois.edu
} 
sets are the boundaries of these cones, and the reset maps are all identity [11]. A distinct feature of a CLS is that it possesses finitely many modes and is subject to state-dependent mode switchings with implicit transition times and implicit mode selection at switching times. Being common in applications, state-dependent switchings complicate many fundamental dynamical and control issues of hybrid systems [11], e.g., the (non)-Zeno and stability analysis.

Among various piecewise linear systems modeled by the CLSs, linear complementarity systems (LCSs) satisfying singleton properties $[33,34]$ have attracted focused attention in recent years. Roughly speaking, an LCS is described by a linear time-invariant ordinary differential equation (ODE) coupled with a linear complementarity problem (LCP) [14]. The LCSs and their generalizations, e.g., differential variational inequalities (DVIs) [29], have received a surge of research interest across several areas, including optimization, systems/control, and robotics, due to their wide applications in the modeling of nonsmooth physical systems and dynamic optimization in operations research. See the two survey articles [8,32] and the research papers [10-12,18,28,29,33-35] as well as the references therein for various issues and results arising from many theoretical and applied problems.

Mode switching is a unique and intrinsic phenomenon in hybrid ODE systems and it plays a critical role in determining fundamental solution properties, e.g., well-posedness, and understanding control-theoretical issues such as stability and observability as well as the numerical analysis of a hybrid system. Stimulated by the modeling and control of complex dynamical systems subject to constraints and possessing multiple subsystems, extensive efforts have been devoted recently to investigating dynamical properties under various mode switching conditions, e.g., [19,24]. For hybrid systems subject to state-dependent mode switchings, the Zeno behavior, which refers to the existence of infinitely many mode switches in finite time, is one of the most crucial switching properties for short-time or finite-time dynamic analysis. It has been shown in [11] that the CLSs are free of Zeno states and hence possess piecewise analytic state trajectories. Additional non-Zeno results have also been obtained for the LCSs satisfying certain singleton properties [33,34], the strongly regular nonlinear complementarity systems [28], and a class of DVIs [17]. In spite of the advances in non-Zeno analysis, much less is known about the mode switching behavior over infinite time domain, which we refer to as the long-time switching behavior throughout the paper. Motivated by the global and long-time dynamic analysis of the CLSs and related systems, for example, stability and long-time observability analysis, the present paper investigates long-time mode switching and stability properties of the CLSs. In particular, long-time switching properties are characterized for several important classes of the CLSs, either expressed in terms of attracting cones or described by finitely verifiable algebraic conditions. By exploiting the switching results and other analytic properties of the CLSs, stability analysis is performed and new stability results are obtained. Compared with the existing stability results for hybrid/switched systems, e.g., [19,20,23,24], the current paper focuses on the CLSs, a class of switched linear systems subject to state-dependent mode switchings, by fully exploring the conic subdivision structure of the systems [15,31]. In the recent paper [1], Arapostathis and Broucke address stability and controllability of planar conewise linear systems with discontinuous right-hand sides. While their characterization of such a CLS is rather complete, their techniques are restricted to planar geometry. It is unclear at this stage whether these techniques can be extended to the general CLS on $\mathbb{R}^{n}$, which is the main concern of our work.

The rest of the paper is organized as follows. In Section 2, mode switching is defined and the "simple switching property" of the CLSs, which pertains to the persistence of certain index sets corresponding to both the forwardtime and the backward-time trajectories, is proved. In Section 3, the notions of attracting cones and global attracting cones are introduced for each mode of a CLS. With the help of these two cones, long-time invariant behaviors of the CLS are investigated. Sections 4 and 5 address the CLSs with infinite mode switchings and the switch-free CLSs respectively; explicit verifiable algebraic conditions are derived to characterize these two classes of CLSs. By linking asymptotic stability to uniform asymptotic stability, the equivalence of asymptotic 
and exponential stability for the CLS is established in Section 6.2; necessary and sufficient Lyapunov stability conditions for switch-free CLSs are given in Section 6.3 via the switching results obtained in Section 5.

\section{Mode SWitchings of CONEWise Linear Systems}

A conewise linear system (CLS) on $\mathbb{R}^{n}$ is given by:

$$
\dot{x}=A_{i} x, \quad \forall x \in \mathcal{X}_{i}=\left\{x \mid C_{i} x \geq 0\right\}, \quad i=1, \ldots, m,
$$

where $A_{i} \in \mathbb{R}^{n \times n}$ and $C_{i} \in \mathbb{R}^{\ell_{i} \times n}$. The family of all the cones $\mathcal{X}_{i}$ forms a conic subdivision of $\mathbb{R}^{n}$ [31], and the right-hand side of the ODE satisfies the following continuity condition: $x \in \mathcal{X}_{i} \cap \mathcal{X}_{j} \Rightarrow A_{i} x=A_{j} x$. Recall that a conic subdivision of $\mathbb{R}^{n}$ possesses the following properties:

(a) the union of all the cones is equal to $\mathbb{R}^{n}$, i.e., $\bigcup_{i=1}^{m} \mathcal{X}_{i}=\mathbb{R}^{n}$;

(b) each cone is solid, i.e., it has a nonempty interior (thus is of dimension $n$ ); and

(c) the intersection of any two cones is either empty or a common proper face of both cones, i.e., $\mathcal{X}_{i} \cap \mathcal{X}_{j}=$ $\mathcal{X}_{i} \cap\left\{x \mid\left(C_{i} x\right)_{\alpha}=0\right\}=\mathcal{X}_{j} \cap\left\{x \mid\left(C_{j} x\right)_{\beta}=0\right\}$ for nonempty index sets $\alpha$ and $\beta$.

The right-hand side of (2.1) is continuous and piecewise linear in $x$, and thus is globally Lipschitz in $x$. Hence it follows that (2.1) has a unique $\mathrm{C}^{1}$ state trajectory, denoted by $x\left(t, x^{0}\right)$, for any initial state $x^{0}$ and all $t$. Without loss of generality, we assume throughout the paper that each matrix $C_{i}$ has no zero rows. Due to this assumption and the fact that $\mathcal{X}_{i}$ is of full dimension, we see that for each index $k=1, \ldots, \ell_{i}$, there exists a vector $\widehat{x}^{k} \in \mathcal{X}_{i}$ such that $\left(C_{i} \widehat{x}^{k}\right)_{k}>0$. Therefore, we must have

$$
\emptyset \neq \operatorname{int} \mathcal{X}_{i}=\left\{x \mid C_{i} x>0\right\}
$$

where int denotes the interior of a set. By (c) of the conic subdivision, it further follows that $\mathcal{X}_{j} \cap \operatorname{int} \mathcal{X}_{i}=\emptyset$ for all $i \neq j$. Let bd $\mathcal{X}_{i} \equiv \mathcal{X}_{i} \backslash \operatorname{int} \mathcal{X}_{i}=\left\{x \in \mathcal{X}_{i} \mid\left(C_{i} x\right)_{k}=0\right.$ for some $\left.k\right\}$ be the boundary of $\mathcal{X}_{i}$.

Associated with the "forward-time" system (2.1) is a backward-time (or reverse-time) system that allows us to obtain reverse-time results easily from a forward-time analysis. Specifically, for a given terminal time $T>0$, define $x^{r}(t) \equiv x(T-t)$. We have $x^{r}(0)=x(T)$ and

$$
\dot{x}^{r}=\widetilde{A}_{i} x^{r}, \quad \text { if } x^{r} \in \mathcal{X}_{i},
$$

where $\widetilde{A}_{i} \equiv-A_{i}$. The latter system remains a CLS. The reverse-time system can be used to derive backwardtime results pertaining to the forward-time trajectory. In particular, the reverse-time CLS has a unique state trajectory for all times and any initial condition.

Definition 2.1. Let $x\left(t, x^{0}\right)$ be a state trajectory of the CLS (2.1) with initial state $x^{0}$, i.e., $x\left(0, x^{0}\right)=x^{0}$. We say that a time instant $t_{*} \geq 0$ is not a switching time along $x\left(t, x^{0}\right)$ if there exist $i \in\{1, \ldots, m\}$ and $\varepsilon>0$ such that $x\left(t, x^{0}\right) \in \mathcal{X}_{i}, \forall t \in\left[t_{*}-\varepsilon, t_{*}+\varepsilon\right]$; otherwise, we say that $t_{*}$ is a switching time along $x\left(t, x^{0}\right)$, and that the CLS has a mode transition or mode switching along $x\left(t, x^{0}\right)$ at $t_{*}$.

We recall some related notation and results on mode switchings from [11] to be used in the subsequent development. An ordered real $\ell$-tuple $a=\left(a_{1}, \ldots, a_{\ell}\right)$ is called lexicographically nonnegative if either $a=0$ or its first nonzero element (from the left) is positive and we write $a \succcurlyeq 0$. An $n$-dimensional vector tuple $\left(x^{1}, \ldots, x^{\ell}\right)$ is called lexicographically nonnegative if each real tuple $\left(x_{i}^{1}, \ldots, x_{i}^{\ell}\right)$ is lexicographically nonnegative for all $i=$ $1, \ldots, n$, and we write $\left(x^{1}, \ldots, x^{\ell}\right) \succcurlyeq 0$. For each $i=1, \ldots, m$, let $\mathcal{Y}_{i} \equiv\left\{x \in \mathbb{R}^{n} \mid\left(C_{i} x, C_{i} A_{i} x, \ldots, C_{i} A_{i}^{n-1} x\right) \succcurlyeq 0\right\}$ be the semiobservable cone associated with the pair $\left(C_{i}, A_{i}\right)$. It is known that for any $x^{0}, x\left(t, x^{0}\right) \in \mathcal{X}_{i}$ for all 


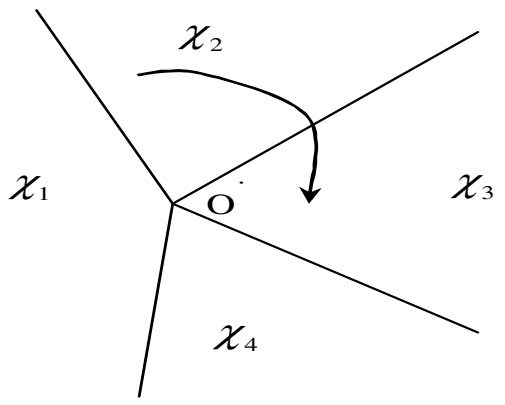

Figure 1. A mode switching.

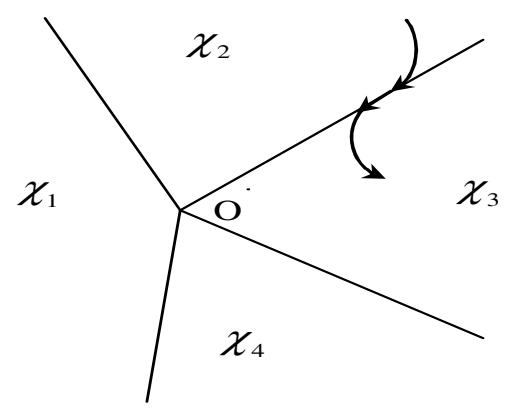

Figure 2. Non-switching ruled out by Proposition 2.2.

$t \geq 0$ sufficiently small if and only if $x^{0} \in \mathcal{Y}_{i}$ [11]. For the given pair $\left(C_{i}, A_{i}\right)$, let $\bar{O}\left(C_{i}, A_{i}\right)$ be its unobservable subspace, namely, $\bar{O}\left(C_{i}, A_{i}\right)=\left\{x \in \mathbb{R}^{n} \mid C_{i} A_{i}^{k} x=0, k=0, \ldots, n-1\right\}$. Given $\xi \in \mathbb{R}^{n}$, define two index sets $\mathcal{I}(\xi) \equiv\left\{i \mid \xi \in \mathcal{X}_{i}\right\}$ and $\mathcal{J}(\xi) \equiv\left\{i \mid \xi \in \mathcal{Y}_{i}\right\}$. Similarly, we can define $\mathcal{J}^{r}(\xi)$ for the associated reverse-time system. It is shown in [11] that a time $t_{*}>0$ is a switching time along a given trajectory $x\left(t, x^{0}\right)$ if and only if $\mathcal{J}\left(x\left(t_{*}, x^{0}\right)\right) \cap \mathcal{J}^{r}\left(x\left(t_{*}, x^{0}\right)\right)=\emptyset$. In [11], Example 3.12, a CLS is given with $\mathcal{J}\left(x\left(t_{*}, x^{0}\right)\right) \neq \mathcal{J}^{r}\left(x\left(t_{*}, x^{0}\right)\right)$ at a non-switching time $t_{*}$. However, this example does not satisfy the proper face property of the conic subdivision. The following proposition shows that the equality $\mathcal{J}\left(x\left(t_{*}, x^{0}\right)\right)=\mathcal{J}^{r}\left(x\left(t_{*}, x^{0}\right)\right)$ holds at any non-switching time $t_{*}$ under the proper face condition. Thus at a non-switching time $t_{*}$, if the forward-time trajectory starting from $x\left(t_{*}, x^{0}\right)$ stays in a cone $\mathcal{X}_{i}$ for a while, then the reverse-time trajectory starting from the same state must also remain in $\mathcal{X}_{i}$ for some time. This property, referred to as the simple switching property, is illustrated in Figure 2; Figure 1 shows a mode switch. Incidentally, while being intuitive, the proof of the simple switching property is not totally trivial as seen below. More importantly, this property is critical to several subsequent results; see Propositions 3.6, 4.1 and Lemma 5.1, the latter being instrumental in proving Proposition 5.2.

Proposition 2.2. For any trajectory $x\left(t, x^{0}\right)$ of the CLS (2.1), a time $t_{*}>0$ is a non-switching time along $x\left(t, x^{0}\right)$ if and only if $\mathcal{J}\left(x\left(t_{*}, x^{0}\right)\right)=\mathcal{J}^{r}\left(x\left(t_{*}, x^{0}\right)\right)$.

Proof. For notational simplicity, let $x^{*}=x\left(t_{*}, x^{0}\right)$. The "if" part follows readily from the facts that $\mathcal{J}\left(x\left(t, x^{0}\right)\right)$ is nonempty for all $t \geq 0$ and that $t_{*}$ is a non-switching time if and only if $\mathcal{J}\left(x^{*}\right) \cap \mathcal{J}^{r}\left(x^{*}\right) \neq \emptyset$. In the next, we show the "only if" part using the proper face property of the conic subdivision. Since $t_{*}$ is a nonswitching time, there is a $j \in \mathcal{J}\left(x^{*}\right) \cap \mathcal{J}^{r}\left(x^{*}\right)$. We claim that $\mathcal{J}^{r}\left(x^{*}\right) \subseteq \mathcal{J}\left(x^{*}\right)$. Suppose not. Then there exists an $i \in \mathcal{J}^{r}\left(x^{*}\right)$ but $i \notin \mathcal{J}\left(x^{*}\right)$. Hence, $i \neq j$. By the choice of $j$, we deduce the existence of $\varepsilon>0$ 
such that $x\left(t, x^{0}\right)=\mathrm{e}^{A_{j}\left(t-t_{0}\right)} x\left(t_{0}, x^{0}\right)$ for all $t \in\left[t_{*}-\varepsilon, t_{*}+\varepsilon\right]$, where $t_{0}=t_{*}-\varepsilon$. Since $x\left(t, x^{0}\right) \in \mathcal{X}_{i} \cap \mathcal{X}_{j}$ for $t \in\left[t_{*}-\varepsilon, t_{*}\right]$, by the proper face condition, there exist two nonempty index sets $\alpha$ and $\beta$ such that $\mathcal{X}_{i} \cap \mathcal{X}_{j}=\mathcal{X}_{i} \cap\left\{x \mid\left(C_{i} x\right)_{\alpha}=0\right\}=\mathcal{X}_{j} \cap\left\{x \mid\left(C_{j} x\right)_{\beta}=0\right\}$; thus $\left(C_{i} x\left(t, x^{0}\right)\right)_{\alpha}=0$ and $\left(C_{j} x\left(t, x^{0}\right)\right)_{\beta}=0$ for all $t \in\left[t_{*}-\varepsilon, t_{*}\right]$. Notice that $\left(C_{j} x\left(t, x^{0}\right)\right)_{\beta}=0, \forall t \in\left[t_{*}-\varepsilon, t_{*}\right]$ implies that $x^{*} \in \bar{O}\left(\left(C_{j}\right)_{\beta \bullet}, A_{j}\right)$. Thus $\left(C_{j} x\left(t, x^{0}\right)\right)_{\beta}=0$ for all $t \in\left[t_{*}, t_{*}+\varepsilon\right]$, which yields $x\left(t, x^{0}\right) \in \mathcal{X}_{j} \cap\left\{x \mid\left(C_{j} x\right)_{\beta}=0\right\}$ for all $t \in\left[t_{*}, t_{*}+\varepsilon\right]$. Consequently, $x\left(t, x^{0}\right) \in \mathcal{X}_{i} \cap\left\{x \mid\left(C_{i} x\right)_{\alpha}=0\right\} \subseteq \mathcal{X}_{i}$ for all $t \in\left[t_{*}, t_{*}+\varepsilon\right]$. However, this is a contradiction to the assumption that $i \notin \mathcal{J}\left(x^{*}\right)$. Therefore, the claim holds. By the similar argument for the reverse-time system, we conclude that $\mathcal{J}^{r}\left(x^{*}\right) \supseteq \mathcal{J}\left(x^{*}\right)$. This thus shows that $\mathcal{J}\left(x^{*}\right)=\mathcal{J}^{r}\left(x^{*}\right)$.

\section{Attracting Cones And LOng-time mode sWitching Properties}

In this section, we study long-time mode switching behaviors of CLS trajectories. It is shown below that such switching behaviors are critically dependent on two attracting cones associated with each mode. We begin by introducing a key positively invariant cone corresponding to a given mode. It is easy to verify that the set $\mathcal{A}_{i}:=\left\{x \mid C_{i} \mathrm{e}^{A_{i} t} x \geq 0, \forall t \in[0, \infty)\right\} \subseteq \mathcal{X}_{i}$ is a closed convex cone. Formally, we call $\mathcal{A}_{i}$ the attracting cone of $\mathcal{X}_{i}$. We collect a few basic facts about the attracting cones as follows.

Lemma 3.1. Let $\mathcal{A}_{i}$ be the attracting cone of $\mathcal{X}_{i}$. Then the following statements hold:

(a) $x \in \mathcal{A}_{i}$ if and only if $\mathrm{e}^{A_{i} t} x \in \mathcal{A}_{i}, \forall t \in[0, \infty)$;

(b) if $x \in \mathcal{A}_{i}$, then $A_{i} x \in \mathcal{T}\left(x ; \mathcal{A}_{i}\right)$, where $\mathcal{T}\left(x ; \mathcal{A}_{i}\right)$ denotes the tangent cone of $\mathcal{A}_{i}$ at $x$;

(c) $\operatorname{lin} \mathcal{A}_{i}=\bar{O}\left(C_{i}, A_{i}\right)$, where $\operatorname{lin} \mathcal{A}_{i}:=\mathcal{A}_{i} \cap\left(-\mathcal{A}_{i}\right)$ is the lineality space of $\mathcal{A}_{i}$.

Thus, $\mathcal{A}_{i}$ is pointed, i.e., $\operatorname{lin} \mathcal{A}_{i}=\{0\}$, if and only if $\bar{O}\left(C_{i}, A_{i}\right)=\{0\}$.

Proof. (a) If $\mathrm{e}^{A_{i} t} x \in \mathcal{A}_{i}$ for all $t \geq 0$, then take $t=0$, we have $x \in \mathcal{A}_{i}$. Conversely, consider $x \in \mathcal{A}_{i}$. For any $t_{*} \geq 0, C_{i} \mathrm{e}^{A_{i} t}\left(\mathrm{e}^{A_{i} t_{*}} x\right)=C_{i} \mathrm{e}^{A_{i}\left(t+t^{*}\right)} x \geq 0$ for all $t \geq 0$ by the definition of $\mathcal{A}_{i}$. Hence, $\mathrm{e}^{A_{i} t_{*}} x \in \mathcal{A}_{i}$.

(b) Let $x \in \mathcal{A}_{i}$ and let $\left\{t_{j}\right\}$ be a positive time sequence converging to zero. Note that $\mathrm{e}^{A_{i} t_{j}} x \in \mathcal{A}_{i}$ due to statement (a) and the vector sequence $\left\{\mathrm{e}^{A_{i} t_{j}} x\right\}$ converges to $x$. Hence, $\lim _{j \rightarrow \infty} \frac{\mathrm{e}^{A_{i} t_{j}} x-x}{t_{j}}=\lim _{j \rightarrow \infty} \frac{\mathrm{e}^{A_{i} t_{j}}-I}{t_{j}} x=$ $A_{i} x$. This shows that $A_{i} x \in \mathcal{T}\left(x ; \mathcal{A}_{i}\right)$.

(c) It is easy to observe that $x \in \mathcal{A}_{i} \cap\left(-\mathcal{A}_{i}\right)$ if and only if $C_{i} \mathrm{e}^{A_{i} t} x=0, \forall t \geq 0$. The latter condition is further equivalent to $x \in \bar{O}\left(C_{i}, A_{i}\right)$. Hence, $\mathcal{A}_{i} \cap\left(-\mathcal{A}_{i}\right)=\{0\}$ if and only if $\bar{O}\left(C_{i}, A_{i}\right)=\{0\}$.

A further characterization of the attracting cone for a given pair $\left(C_{i}, A_{i}\right)$ seems difficult. For one thing, such a cone is usually non-polyhedral. As an example, consider the pair

$$
C_{i}=\left[\begin{array}{lll}
1 & 0 & 0
\end{array}\right], \quad A_{i}=\left[\begin{array}{lll}
0 & 1 & 0 \\
0 & 0 & 1 \\
0 & 0 & 0
\end{array}\right] .
$$

The associated cone $\mathcal{A}_{i}$ is the non-polyhedral cone $\left\{\left(x_{1}, x_{2}, x_{3}\right) \in \mathbb{R}^{3} \mid x_{1} \geq 0, x_{3} \geq 0, x_{2} \geq-\sqrt{2 x_{1} x_{3}}\right\}$. In general, if $A_{i}$ has the zero eigenvalue only, then characterizing its attracting cone can be formulated as a semialgebraic problem; see Section 4.1.1. Despite this difficulty, the next theorem provides a useful result that relates the non-triviality of $\mathcal{A}_{i}$ to a constrained eigenvector property of $A_{i}$ on $\mathcal{X}_{i}$; its proof follows from [27], Lemma 1, based on a fixed point argument.

Theorem 3.2. $\mathcal{A}_{i} \neq\{0\}$ if and only if either $\bar{O}\left(C_{i}, A_{i}\right) \neq\{0\}$ or the matrix $A_{i}$ has a real eigenvalue with an associated eigenvector in $\mathcal{X}_{i}$, i.e., there exists $0 \neq v \in \mathcal{X}_{i}$ such that $A_{i} v=\lambda v$ for some $\lambda \in \mathbb{R}$. 
An interpretation of Theorem 3.2 in term of mode switchings is given in Remark 4.9. This theorem can be used to characterize cone observability [4]. Specifically, consider a closed convex cone $\mathcal{K}$ and a linear system associated with $A \in \mathbb{R}^{n \times n}$ and $C \in \mathbb{R}^{m \times n}$ :

$$
\dot{x}=A x, \quad y=C x,
$$

and define the set $\mathcal{A}(A, C, \mathcal{K}):=\left\{x \mid C \mathrm{e}^{A t} x \in \mathcal{K}, \forall t \geq 0\right\}$. The system (3.1) is $\mathcal{K}$-observable if $\mathcal{A}(A, C, \mathcal{K})=\{0\}$ [4], Definition 3.1 of Chapter 8. Obviously Theorem 3.2, together with (c) of Lemma 3.1, gives a necessary and sufficient condition for $\mathbb{R}_{+}^{m}$-observability of (3.1). The following corollary extends the latter remark to a general closed convex cone $\mathcal{K}$; the proof is similar to Theorem 3.2 and hence omitted.

Corollary 3.3. Let $\mathcal{K}$ be a closed convex cone whose lineality space is the null space of a matrix $M$, i.e., $\mathcal{K} \cap(-\mathcal{K})=\operatorname{ker} M$. Then the linear system $(3.1)$ is $\mathcal{K}$-observable if and only if the following two conditions hold: (a) $\bar{O}(M C, A)=\{0\}$ and (b) there is no real eigenvector $v$ of $A$ such that $C v \in \mathcal{K}$.

Extending the cone $\mathcal{A}_{i}$, we define the global attracting cone $\widehat{\mathcal{A}_{i}}$ associated with the mode $\mathcal{X}_{i}$ as:

$$
\widehat{\mathcal{A}_{i}}=\left\{v \in \mathbb{R}^{n} \mid \exists t_{*} \geq 0 \text { such that } x(t, v) \in \mathcal{X}_{i}, \forall t \geq t_{*}\right\}
$$

It is clear that $\mathcal{E}_{i} \subseteq \mathcal{A}_{i} \subseteq \widehat{\mathcal{A}_{i}}$, where $\mathcal{E}_{i}:=\left\{x \mid A_{i} x=0, x \in \mathcal{X}_{i}\right\}$ is the set of equilibrium states of the CLS in $\mathcal{X}_{i}$. Unlike $\mathcal{A}_{i}, \widehat{\mathcal{A}_{i}}$ is neither convex nor closed in general. Less obvious is the equality:

$$
\widehat{\mathcal{A}_{i}}=\left\{x^{r}\left(t, x^{0}\right) \mid x^{0} \in \mathcal{A}_{i}, t \geq 0\right\},
$$

which shows that $\widehat{\mathcal{A}}_{i}$ is the set of reachable states of trajectories of the reverse-time CLS (2.3) that begin with initial states in $\mathcal{A}_{i}$. To establish (3.2), let $x^{*}=x^{r}\left(t_{*}, x^{0}\right)$ for some $t_{*} \geq 0$ and $x^{0} \in \mathcal{A}_{i}$. Hence, $x\left(t_{*}, x^{*}\right)=x^{0}$ by the uniqueness of the forward and backward time trajectories. Moreover, $x\left(t, x\left(t_{*}, x^{*}\right)\right)=x\left(t, x^{0}\right)$ for all $t \geq 0$. Since $x^{0} \in \mathcal{A}_{i}$, there exists $t_{0} \geq 0$ such that $x\left(t, x^{0}\right) \in A_{i}$ for all $t \geq t_{0}$. Therefore $x\left(t, x^{*}\right)$ must be in $\mathcal{A}_{i}$ for all $t \geq t_{0}+t_{*}$. This shows $x^{*} \in \widehat{\mathcal{A}}_{i}$. Therefore, the left-hand set in (3.2) is contained in $\widehat{\mathcal{A}}_{i}$. The reverse inclusion is easy. Based on the equality (3.2), it follows readily that the global attracting cone is nontrivial if and only if its corresponding attracting cone is so. Furthermore, it is easy to observe that if $x^{0} \in \operatorname{lin} \mathcal{A}_{i} \equiv \bar{O}\left(C_{i}, A_{i}\right)$, then $x^{r}\left(t, x^{0}\right)=\mathrm{e}^{-A_{i} t} x^{0} \in \mathcal{A}_{i}$ for all $t \geq 0$ by solution uniqueness of the reverse-time CLS. Therefore, if $\mathcal{A}_{i}=\operatorname{lin} \mathcal{A}_{i}$, then we have $\widehat{\mathcal{A}_{i}}=\mathcal{A}_{i}$ by (3.2). These two results are formally stated in the proposition below without a proof.

Proposition 3.4. Let $\widehat{\mathcal{A}_{i}}$ and $\mathcal{A}_{i}$ be the two attracting cones defined above. Then $\widehat{\mathcal{A}_{i}} \neq\{0\}$ if and only if $\mathcal{A}_{i} \neq\{0\}$. Moreover, if $\mathcal{A}_{i}=\operatorname{lin} \mathcal{A}_{i}$, then $\widehat{\mathcal{A}_{i}}=\mathcal{A}_{i}=\operatorname{lin} \mathcal{A}_{i}$.

The next result identifies several important properties of the cone $\widehat{\mathcal{A}_{i}}$. It provides a clue as to why this cone may not be closed, showing in particular that the last switching time $\tau_{*}(v)$ of a state $v \in \widehat{\mathcal{A}_{i}}$, which is defined as:

$$
\tau_{*}(v) \equiv \inf \left\{t_{*} \geq 0 \mid x(t, v) \in \mathcal{X}_{i}, \forall t \geq t_{*}\right\}
$$

plays an important role in the closeness of $\widehat{\mathcal{A}_{i}}$. The result also establishes a special arcwise connected property of $\widehat{\mathcal{A}}_{i}$; see part (c) below. In particular, it shows that any two linearly independent vectors in $\widehat{\mathcal{A}}_{i}$ can be connected by a continuous injective curve in $\widehat{\mathcal{A}_{i}}$ not passing through the origin. (Note that any two points in the cone $\widehat{\mathcal{A}_{i}}$ can be trivially connected by two line segments in $\widehat{\mathcal{A}_{i}}$ through the origin.) 
Proposition 3.5. The following three statements hold for the cone $\widehat{\mathcal{A}_{i}}$.

(a) Let $\left\{v^{k}\right\} \subseteq \widehat{\mathcal{A}_{i}}$ be a sequence converging to $v^{*}$. If $\limsup _{k \rightarrow \infty} \tau_{*}\left(v^{k}\right)<\infty$, then $v^{*} \in \widehat{\mathcal{A}_{i}}$. Thus, $\widehat{\mathcal{A}_{i}}$ is closed if $\tau_{*}(v)$ is bounded on compact subsets of $\widehat{\mathcal{A}_{i}}$.

(b) If $v^{*} \in \widehat{\mathcal{A}_{i}}$ is such that $x\left(t_{*}, v^{*}\right) \in \operatorname{int} \mathcal{A}_{i}$ for a $t_{*} \geq 0$, then $\tau_{*}(v) \leq t_{*}$ for all $v$ sufficiently close to $v^{*}$.

(c) The following two statements are equivalent for any two nonzero vectors $u^{*} \neq v^{*}$ in $\widehat{\mathcal{A}_{i}}$ :

(c1) there is a continuous injective curve $c:[0,1] \rightarrow \widehat{\mathcal{A}_{i}}$ such that $c(0)=u^{*}, c(1)=v^{*}$, and $c(\lambda) \neq 0$ for all $\lambda \in[0,1]$;

(c2) $u^{*}, v^{*}$ and $\mathcal{A}_{i}$ do not satisfy all the following three conditions: $\operatorname{dim}\left(\mathcal{A}_{i}\right)=1, u^{*}, v^{*} \in \operatorname{lin} \mathcal{A}_{i}$, and $u^{*}=-\tau v^{*}$ for a positive scalar $\tau$.

Proof. To prove (a), let $t_{k}:=\tau_{*}\left(v^{k}\right)$. We may assume without loss of generality that $\left\{t_{k}\right\}$ converges to $t_{*} \geq 0$. This implies that for all $k$ sufficiently large, $x\left(t, v^{k}\right) \in \mathcal{X}_{i}, \forall t \geq t_{*}+1$. Since $x(t, \cdot)$ is continuous and $\mathcal{X}_{i}$ is closed, it follows that $x\left(t, v^{*}\right) \in \mathcal{X}_{i}$ for all $t \geq t_{*}+1$, establishing that $v^{*} \in \widehat{\mathcal{A}_{i}}$. The second assertion in (a) does not require further proof.

To show (b), let $x^{*}:=x\left(t_{*}, v^{*}\right) \in \operatorname{int} \mathcal{A}_{i}$. Then there is a closed ball $\mathcal{B} \subseteq \mathcal{A}_{i}$ centered at $x^{*}$. Since $t_{*}$ is finite, by the Lipschitz solution dependence result, we have a ball $\mathcal{B}_{0}$ centered at $v^{*}$ such that for any $v \in \mathcal{B}_{0}$, $x\left(t_{*}, v\right) \in \mathcal{B} \subseteq \mathcal{A}_{i}$. Hence, $x(t, v) \in \mathcal{X}_{i}$ for all $t \geq t_{*} ;$ thus $\tau_{*}(v) \leq t_{*}$.

To prove (c), we first show (c1) $\Rightarrow$ (c2) via contradiction. Suppose (c2) does not hold. We deduce from $0 \neq u^{*} \in \operatorname{lin} \mathcal{A}_{i}$ that $\operatorname{dim}\left(\operatorname{lin} \mathcal{A}_{i}\right) \geq 1$. Since $\operatorname{lin} \mathcal{A}_{i} \subseteq \mathcal{A}_{i}$ and $\operatorname{dim}\left(\mathcal{A}_{i}\right)=1$, we have $\operatorname{dim}\left(\operatorname{lin} \mathcal{A}_{i}\right)=1$ which further implies $\mathcal{A}_{i}=\operatorname{lin} \mathcal{A}_{i}$. By Proposition 3.4, $\widehat{\mathcal{A}_{i}}=\operatorname{lin} \mathcal{A}_{i}$. Hence $\widehat{\mathcal{A}_{i}}=\operatorname{Span}\left\{u^{*}\right\}$ is a one-dimensional subspace of $\mathbb{R}^{n}$, i.e., $\widehat{\mathcal{A}_{i}}$ is a line through the origin parallel to $u^{*}$ and $v^{*}$. Therefore, any continuous curve in $\widehat{\mathcal{A}_{i}}$ connecting $u^{*}$ and $v^{*}$ passes through the origin. This is contradictory to (c1).

To show $(\mathrm{c} 2) \Rightarrow(\mathrm{c} 1)$, we prove the following claim first: for the given $u^{*}$ and $v^{*}$, exactly one of the two statements: (c1) or $\left[u^{*}, v^{*} \in \operatorname{lin} \mathcal{A}_{i}\right.$ and $u^{*}=-\tau v^{*}$ for some $\left.\tau>0\right]$, holds. Let $t_{*} \geq 0$ be such that $x^{*}:=x\left(t_{*}, u^{*}\right) \in \mathcal{A}_{i}$ and $y^{*}:=x\left(t_{*}, v^{*}\right) \in \mathcal{A}_{i}$. Since $u^{*}, v^{*}$ are nonzero, we deduce that $x^{*} \neq 0$ and $y^{*} \neq 0$ by the global Lipschitz property of the CLS. Thanks to the convexity of $\mathcal{A}_{i}, x^{*}+\lambda\left(y^{*}-x^{*}\right) \in \mathcal{A}_{i}$ for all $\lambda \in[0,1]$. Then exactly one of the following two cases holds:

(i) $x^{*}+\lambda\left(y^{*}-x^{*}\right) \neq 0$ for all $\lambda \in[0,1]$. In this case, we define the curve $c:[0,1] \rightarrow \mathbb{R}^{n}$ as $c(\lambda)=$ $x^{r}\left(t_{*}, x^{*}+\lambda\left(y^{*}-x^{*}\right)\right)$. We immediately have $c(0)=x^{r}\left(t_{*}, x^{*}\right)=u^{*}$ and $c(1)=x^{r}\left(t_{*}, y^{*}\right)=v^{*}$. Moreover, $c(\lambda) \neq 0$ for all $\lambda \in[0,1]$ due to $x^{*}+\lambda\left(y^{*}-x^{*}\right) \neq 0$ and the global Lipschitz property of the reverse-time CLS. Besides, it is easy to verify $x\left(t_{*}, c(\lambda)\right)=x^{*}+\lambda\left(y^{*}-x^{*}\right) \in \mathcal{A}_{i}$ for each $\lambda \in[0,1]$. Thus $c([0,1]) \subset \widehat{\mathcal{A}_{i}}$. The curve is continuous because of continuous solution dependence on initial conditions and the solution uniqueness of the reverse-time CLS implies that the curve is one-to-one.

(ii) $x^{*}+\lambda_{*}\left(y^{*}-x^{*}\right)=0$ for some $\lambda_{*} \in(0,1)$. In this case, $x^{*}=-\tau y^{*}$, where $\tau:=\lambda_{*} /\left(1-\lambda_{*}\right)>0$. Since $\mathcal{A}_{i}$ is a cone, both $x^{*} \in \operatorname{lin} \mathcal{A}_{i}$ and $y^{*} \in \operatorname{lin} \mathcal{A}_{i}$. Recalling $\operatorname{lin} \mathcal{A}_{i}=\bar{O}\left(C_{i}, A_{i}\right)$ (see (c) of Lem. 3.1), we obtain $C_{i} \mathrm{e}^{A_{i} t} x^{*} \equiv 0, \forall t \in \mathbb{R}$, i.e., $\mathrm{e}^{A_{i} t} x^{*} \in \mathcal{X}_{i}$ for all $t$. The solution uniqueness of the reverse-time CLS implies that $x^{r}\left(t, x^{*}\right)=\mathrm{e}^{-A_{i} t} x^{*}$ and $x^{r}\left(t, x^{*}\right) \in \operatorname{lin} \mathcal{A}_{i}$ for all $t \geq 0$. Hence, we deduce that $u^{*}=\mathrm{e}^{-A_{i} t_{*}} x^{*} \in \operatorname{lin} \mathcal{A}_{i}$. Similarly, $v^{*}=\mathrm{e}^{-A_{i} t_{*}} y^{*} \in \operatorname{lin} \mathcal{A}_{i}$. Since $x^{*}=-\tau y^{*}$, we have $u^{*}=-\tau v^{*}$. This completes the proof of the claim.

We now return to the proof of (c2) $\Rightarrow(\mathrm{c} 1)$. Suppose that (c2) holds but (c1) does not for the given $u^{*}, v^{*}$. The above claim yields that $u^{*}, v^{*} \in \operatorname{lin} \mathcal{A}_{i} \subseteq \widehat{\mathcal{A}_{i}}$ and $u^{*}=-\tau v^{*}$ for some $\tau>0$. Since (c2) holds, $\operatorname{dim}\left(\mathcal{A}_{i}\right) \neq 1$. Furthermore, $\mathcal{A}_{i} \neq\{0\}$ implies that $\operatorname{dim}\left(\mathcal{A}_{i}\right) \geq 2$. As a result, there exists a nonzero vector $w^{*} \in \mathcal{A}_{i} \subseteq \widehat{\mathcal{A}_{i}}$ such that each of the pairs $\left(u^{*}, w^{*}\right)$ and $\left(w^{*}, v^{*}\right)$ is linearly independent. Applying the claim proved above again, we obtain two continuous injective curves in $\widehat{\mathcal{A}_{i}}$, neither passing through the origin, such that they connect $\left(u^{*}, w^{*}\right)$ and $\left(w^{*}, v^{*}\right)$ respectively. By concatenating the two curves, we have a continuous injective curve in $\widehat{\mathcal{A}_{i}}$ 


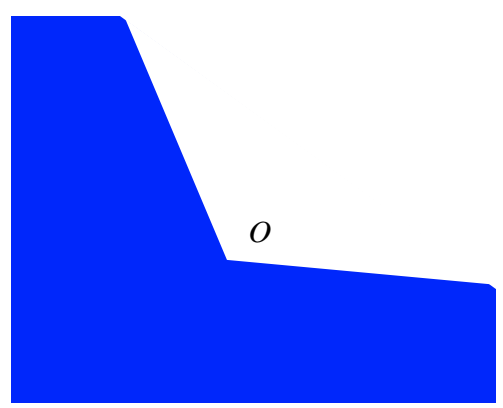

Figure 3. A possible configuration of $\widehat{\mathcal{A}_{i}}$.

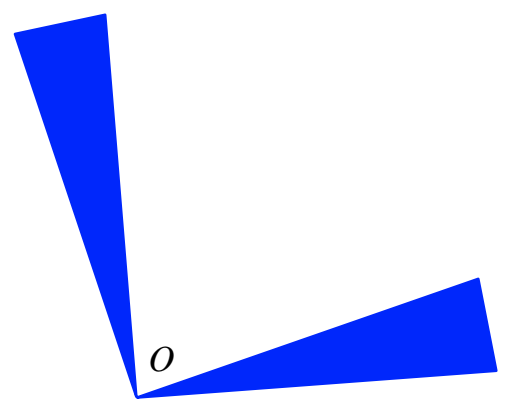

Figure 4. An impossible configuration of $\widehat{\mathcal{A}_{i}}$.

connecting $u^{*}$ and $v^{*}$ without passing through the origin. This is a contradiction to the hypothesis that (c1) does not hold.

The above connectedness result is geometrically appealing and provides additional structure to the cone $\widehat{\mathcal{A}_{i}}$. For example, if $\widehat{\mathcal{A}_{i}} \neq\{0\}$ is such that there is a unique continuous injective curve in $\widehat{\mathcal{A}_{i}}$ connecting any two different points in $\widehat{\mathcal{A}}_{i}$, then $\widehat{\mathcal{A}_{i}}$ is either (i) a linear ray with the origin as its starting point, or (ii) a line through the origin. In the former case, $\widehat{\mathcal{A}_{i}}=\mathcal{A}_{i}$, and $\widehat{\mathcal{A}_{i}}=\mathcal{A}_{i} \cup\left(-\mathcal{A}_{i}\right)$ in the latter. Moreover, if $\widehat{\mathcal{A}_{i}}$ is the union of a family of nontrivial cones $\mathcal{C}_{j}$ satisfying $\mathcal{C}_{j} \cap \mathcal{C}_{k}=\{0\}$ for any $j \neq k$, then for each $j$, lin $\mathcal{A}_{i} \cap \mathcal{C}_{j} \neq\{0\}$ and a cone $\mathcal{C}_{k}$ exists with $j \neq k$ such that $\mathcal{C}_{j} \cap\left(-\mathcal{C}_{k}\right) \neq\{0\}$. See Figures 3 and 4 for illustration of a possible and an impossible configuration of $\widehat{\mathcal{A}_{i}}$ in $\mathbb{R}^{2}$.

The global attracting cone $\widehat{\mathcal{A}}_{i}$ can be used to characterize the CLS with finitely many mode switchings. The proof uses the simple switching property.

Proposition 3.6. Each CLS trajectory has finitely many mode switches in $[0, \infty)$ if and only if $\bigcup_{i=1}^{m} \widehat{\mathcal{A}}_{i}=\mathbb{R}^{n}$.

Proof. For the "if" part, let $x^{0} \in \mathbb{R}^{n}$ be arbitrary. It follows that $x^{0} \in \widehat{\mathcal{A}_{i}}$ for some $i$. Therefore, a time $t_{*} \geq 0$ exists such that $x\left(t, x^{0}\right) \in \mathcal{X}_{i}$ for all $t \geq t_{*}$. This means that there is no mode switching after $t_{*}$. On the other hand, the non-Zeno result in [11] shows that there are finitely many mode switchings along $x\left(t, x^{0}\right)$ on $\left[0, t_{*}\right]$. Hence, every trajectory of the CLS has finitely many mode switches on $[0, \infty)$. Conversely, suppose that the CLS has a finite number of mode switches on $[0, \infty)$ along any trajectory. Then for any given $x^{0} \in \mathbb{R}^{n}$, there is a time $t_{*} \geq 0$ such that any $t \geq t_{*}$ is a non-switching time along $x\left(t, x^{0}\right)$. By Proposition 2.2 , we see that 
the (nonempty) index set $\mathcal{J}\left(x\left(t, x^{0}\right)\right)$ remains invariant for all $t \geq t_{*}$. This shows that $x\left(t, x^{0}\right) \in \mathcal{X}_{i}, \forall t \geq t_{*}$ for some $i \in \mathcal{J}\left(x\left(t_{*}, x^{0}\right)\right)$. Therefore, $x^{0} \in \widehat{\mathcal{A}}_{i}$.

For a CLS such that each trajectory has finitely many mode switchings in infinite (forward) time, the number of mode switchings is generally unbounded for all trajectories, even for the trajectories reaching the same point. This causes difficulty in studying long-time switching behavior of the CLS. To overcome this difficulty, we introduce the "uniform" mode switching notion as follows. We call an $x^{*} \in \mathcal{A}_{i}$ a regular attracting point if a positive integer $N\left(x^{*}\right)$ exists such that for each trajectory $x(t, v)$ with $x^{*}:=x(s, v)$ for some $s \geq 0$, the number of mode switches along $x(t, v)$ on $[0, \infty)$ is less than or equal to $N\left(x^{*}\right)$. The following result characterizes such points.

Proposition 3.7. Let $\widehat{\mathcal{A}_{i}^{r}}$ be the global attracting cone of $\mathcal{X}_{i}$ defined for the reverse-time CLS. Then $x^{*} \in \mathcal{A}_{i}$ is a regular attracting point if and only if $x^{*} \in \widehat{\mathcal{A}_{j}^{r}}$ for some $j$ (possibly different from $i$ ).

Proof. To show the "if" part, we first observe that if $x^{*} \in \widehat{\mathcal{A}}_{j}^{r}$, then $t_{r} \geq 0$ exists such that $x^{r}\left(t, x^{*}\right) \in \mathcal{A}_{j}^{r}$ for all $t \geq t_{r}$. The non-Zenoness of the CLS thus implies that there are $N$ mode switchings along $x^{r}\left(t, x^{*}\right)$ on $\left[0, t_{r}\right]$, where $N$ is a nonnegative integer. By the switching property given in Proposition 2.2 , we see that if $\widehat{x}$ is a switching state on a forward-time trajectory, so is on the same trajectory but in the backward-time direction. Hence for any $v$ such that $x(t, v)$ reaches $x^{*}$ in the forward time, there are at most $N$ mode switchings along $x(t, v)$. This completes the proof for this part. We next prove the "only if" part via contradiction, i.e., suppose $x^{*} \notin \bigcup_{j=1}^{m} \widehat{\mathcal{A}_{j}^{r}}$. Thus $x^{r}\left(t, x^{*}\right)$ has infinitely many mode switchings on $[0, \infty)$ (since otherwise $x^{r}\left(t, x^{*}\right)$ will remain in one piece for all $t$ sufficiently large, which leads to a contradiction). That is, for any positive number $M$, there is a $t_{M} \geq 0$ such that there are $M$ mode switchings along $x^{r}(t, v)$ on $\left[0, t_{M}\right]$. Letting $v_{M}=x^{r}\left(t_{M}, v\right)$, we see that $x\left(t, v_{M}\right)$ has $M$ mode switchings on $\left[0, t_{M}\right]$ before it reaches the non-switching state $x^{*}$. Since $M$ is arbitrarily large, we conclude that the number of mode switchings of all the trajectories reaching $x^{*}$ in the forward time is unbounded. This is a contradiction.

We say that a CLS satisfies the uniform finite switching property if (i) any (forward-time) trajectory of the CLS has finitely many mode switchings in infinite time, and (ii) each $\mathcal{A}_{i}$ contains regular attracting points only. The following result is easy to obtain via Proposition 3.7: the CLS (2.1) satisfies the uniform finite switching property if and only if $\bigcup_{i=1}^{m} \widehat{\mathcal{A}_{i}}=\mathbb{R}^{n}$ and $\bigcup_{i=1}^{m} \widehat{\mathcal{A}}_{i}^{r}=\mathbb{R}^{n}$. This result may be served as a necessary condition for a CLS with a bound on the number of mode switchings of all trajectories. However, instead of pursuing further general discussion, we address two special classes of the CLSs in the next two sections by obtaining explicit characterization conditions for these CLSs.

\section{CLSs With INFINITE MOdE SWITCHINGS}

In this section, we focus on the CLSs satisfying (b) of Proposition 4.1 below, which are referred to as the CLSs with infinite mode switchings. To characterize such CLSs, we only need to consider trajectories starting from non-equilibrium states when dealing with those with at least one switching.

Proposition 4.1. Let $\mathcal{E}:=\bigcup_{i=1}^{m} \mathcal{E}_{i}$ be the equilibrium set of the $C L S$. Then $\mathcal{X}_{i} \cap \mathcal{E}=\mathcal{E}_{i}$ for all $i=1, \ldots, m$ and the following four statements are equivalent:

(a) for any $x^{0} \notin \mathcal{E}$, the trajectory $x\left(\cdot, x^{0}\right)$ has a mode switching in $[0, \infty)$;

(b) for any $x^{0} \notin \mathcal{E}$, the trajectory $x\left(\cdot, x^{0}\right)$ has infinitely many mode switchings in $[0, \infty)$; 
(c) for each $i=1, \ldots, m$ and any $x^{0} \in \mathcal{Y}_{i} \backslash \mathcal{E}_{i}$, there exists a $t_{*}>0$ such that $\mathrm{e}^{A_{i} t_{*}} x^{0} \notin \mathcal{X}_{i}$;

(d) $\widehat{\mathcal{A}_{i}}=\mathcal{A}_{i}=\mathcal{E}_{i}=\mathcal{X}_{i} \cap \mathcal{E}$ for all $i=1, \ldots, m$.

Moreover, if any of (a)-(d) holds, then $\bar{O}\left(C_{i}, A_{i}\right)=\operatorname{ker}\left[\begin{array}{c}C_{i} \\ A_{i}\end{array}\right]$ for all $i=1, \ldots, m$.

Proof. Clearly, $\mathcal{E}_{i} \subseteq \mathcal{X}_{i} \cap \mathcal{E}$. Conversely, if $x \in \mathcal{X}_{i} \cap \mathcal{E}_{j}$ for some $j \neq i$, then $x \in \mathcal{X}_{i} \cap \mathcal{X}_{j}$. Thus, $A_{i} x=A_{j} x=0$; hence $x \in \mathcal{E}_{i}$. Therefore, $\mathcal{E}_{i}=\mathcal{X}_{i} \cap \mathcal{E}$.

(a) $\Leftrightarrow$ (b). We only need to show (a) $\Rightarrow$ (b). Let $x^{0} \notin \mathcal{E}$. Suppose for contradiction that $x\left(\cdot, x^{0}\right)$ has finitely many switchings on $[0, \infty)$. Let $t_{*} \equiv \tau_{*}\left(x^{0}\right)$ be the last switching time defined in (3.3). Thus the trajectory starting from $x\left(t_{*}+1, x^{0}\right)$ has no switching in the forward time. By (a), $x\left(t_{*}+1, x^{0}\right)$ must be in $\mathcal{E}$. This shows that $x\left(t_{*}+1, x^{0}\right)$ is an equilibrium of the CLS. We deduce, via the solution uniqueness of the reverse-time trajectory, that $x\left(t, x^{0}\right)$ is an equilibrium for all $t \geq 0$. This is a contradiction to the assumption $x^{0} \notin \mathcal{E}$. Hence, (a) $\Leftrightarrow$ (b).

(a) $\Leftrightarrow$ (c). We show (a) $\Rightarrow$ (c) first. Clearly, if (c) fails, then there exists an $x^{0} \in \mathcal{Y}_{i} \backslash \mathcal{E}_{i}$ such that $x\left(t, x^{0}\right) \equiv \mathrm{e}^{A_{i} t} x^{0} \in \mathcal{X}_{i}$ for all $t \geq 0$. Hence no switching occurs along $x\left(t, x^{0}\right)$, which is a contradiction since $x^{0} \notin \mathcal{E}$. To show the reverse, recall that for any $x^{0}, x^{0} \in \mathcal{Y}_{i}$ for some $i \in\{1, \ldots, m\}$. This implies that $x\left(t, x^{0}\right) \in \mathcal{X}_{i}$ for all $t \geq 0$ sufficiently small. If $x^{0} \notin \mathcal{E}$, then $x^{0} \in \mathcal{Y}_{i} \backslash \mathcal{E}_{i}$. Thus (c) yields the existence of a time instant $\widehat{t} \in\left(0, t_{*}\right)$ such that $x\left(t, x^{0}\right) \in \mathcal{X}_{i}, \forall t \in[0, \widehat{t}]$ and that $i \notin \mathcal{J}\left(x\left(\widehat{t}, x^{0}\right)\right)$, where the former condition further shows that $i \in \mathcal{J}^{r}\left(x\left(\widehat{t}, x^{0}\right)\right)$. Hence, we deduce via the simple switching property proven in Proposition 2.2 that $x\left(t, x^{0}\right)$ has a mode switching at $\widehat{t}$. This leads to (a).

(a) $\Leftrightarrow$ (d). We first show that (a) implies $\mathcal{A}_{i}=\mathcal{E} \cap \mathcal{X}_{i}$ for all $i=1, \ldots, m$. Note that under (a), for any $x^{0} \in \mathcal{X}_{i} \backslash \mathcal{E}$, there exists $t_{*}>0$ such that $\mathrm{e}^{A_{i} t_{*}} x^{0} \notin \mathcal{X}_{i}$ (otherwise there is no switching along $x\left(t, x^{*}\right)$ ). Hence $x^{0} \notin \mathcal{A}_{i}$. Therefore, $\mathcal{A}_{i} \subseteq \mathcal{E} \cap \mathcal{X}_{i}$; the other direction is trivial. Similarly, one can show, via the equivalence of (a) and (b), that if $x^{0} \notin \mathcal{E}$, then $x^{0} \notin \bigcup_{i=1}^{m} \widehat{\mathcal{A}_{i}}$. Thus $\widehat{\mathcal{A}_{i}} \subseteq \mathcal{E}$ for all $i$. Moreover, if $x^{0} \in \mathcal{E}$ but not in $\mathcal{X}_{i}$, $x\left(t, x^{0}\right) \equiv x^{0}, \forall t$ will never be in $\mathcal{X}_{i}$ for all $t \geq 0$. Thus such $x^{0}$ is not in $\widehat{\mathcal{A}_{i}}$, which implies $\widehat{\mathcal{A}_{i}} \subseteq \mathcal{E} \cap \mathcal{X}_{i}$ for each $i$. The reverse inclusion holds trivially. Therefore, (a) implies (d). Conversely, if (d) holds, then for a given $x^{0} \notin \mathcal{E}, x^{0} \notin \bigcup_{i=1}^{m} \mathcal{A}_{i}$. Hence for any $i \in \mathcal{J}\left(x^{0}\right)$ (i.e., $\left.x^{0} \in \mathcal{Y}_{i}\right)$, there exists $t_{*}>0$ such that $\mathrm{e}^{A_{i} t_{*}} x^{0} \notin \mathcal{X}_{i}$. This shows the existence of one switching along the trajectory $x\left(t, x^{0}\right)$ via the previous argument for (c) $\Rightarrow$ (a). Consequently, (a) holds true.

Finally, if any one of conditions (a)-(d) holds, then $\bar{O}\left(C_{i}, A_{i}\right)=\mathcal{A}_{i} \cap\left(-\mathcal{A}_{i}\right)=\mathcal{E}_{i} \cap\left(-\mathcal{E}_{i}\right)$, from which the equality $\bar{O}\left(C_{i}, A_{i}\right)=\operatorname{ker}\left[\begin{array}{c}C_{i} \\ A_{i}\end{array}\right]$ follows readily.

\subsection{Further necessary and sufficient conditions}

In this section, we derive verifiable conditions that characterize the CLSs with infinitely many mode switchings. In view of (c) and (d) of Proposition 4.1, it suffices to treat each pair $\left(C_{i}, A_{i}\right)$ individually. For notational simplicity, we drop the subscript in this pair and use $(C, A)$ throughout this subsection. We also assume without loss of generality that $A$ is in real Jordan canonical form via a (real) similar transformation. Let $A$ have $p$ distinct real eigenvalues $\lambda_{i}$ and $s$ distinct complex eigenvalue pairs $\mu_{j}$ and $\bar{\mu}_{j}$. Hence, $A$ has the following Jordan form [13], Section 5.6,

$$
A=\left[\begin{array}{llll}
J_{1} & & & \\
& J_{2} & & \\
& & \ddots & \\
& & & J_{p+s}
\end{array}\right]
$$


where $J_{i}$ denotes all the Jordan blocks associated with a real eigenvalue $\lambda_{i}$ or a complex eigenvalue and its conjugate $\left(i . e . \mu_{i}\right.$ and $\bar{\mu}_{j}$ ). (We assume that $J_{1}, \ldots, J_{p}$ correspond to the distinct real eigenvalues and $J_{p+1}, \ldots, J_{p+s}$ correspond to the distinct complex eigenvalue pairs.) Specifically, $J_{i}$ is given by

$$
J_{i}=\left[\begin{array}{llll}
J_{i 1} & & & \\
& J_{i 2} & & \\
& & \ddots & \\
& & & J_{i r(i)}
\end{array}\right] .
$$

Here $r(i)$ is the number of Jordan blocks in $J_{i}$ and $J_{i j}$ is the $j$ th (real) Jordan block given by

(i) $J_{i j}=\left[\begin{array}{ccccc}\lambda_{i} & 1 & & & \\ & \lambda_{i} & 1 & & \\ & & \ddots & \ddots & \\ & & \ddots & 1 \\ & & & & \lambda_{i}\end{array}\right]$ corresponding to the real eigenvalue $\lambda_{i}$; or
(ii) $J_{i j}=\left[\begin{array}{ccccc}D_{i} & I_{2} & & & \\ & D_{i} & I_{2} & & \\ & & \ddots & \ddots & \\ & & & \ddots & I_{2} \\ & & & & D_{i}\end{array}\right]$ corresponding to the complex eigenvalue pair $\sigma_{i} \pm \imath \omega_{i}$, where
$\sigma_{i}, \omega_{i} \in \mathbb{R}$ with $\omega_{i}>0, I_{2}$ is the $2 \times 2$ identity matrix and $D_{i}=\left[\begin{array}{cc}\sigma_{i} & \omega_{i} \\ -\omega_{i} & \sigma_{i}\end{array}\right]$. Accordingly, the matrix $C$ can be written as

$$
C=\left[\begin{array}{llll}
C_{1} & C_{2} & \ldots & C_{p+s}
\end{array}\right], \quad \text { with } \quad C_{i}=\left[\begin{array}{llll}
C_{i 1} & C_{i 2} & \ldots & C_{i r(i)}
\end{array}\right],
$$

where $C_{i j}=\left[C_{i j \ell}\right]_{\ell=1}^{k_{i j}}$ and $C_{i j \ell}$ represents the $\ell$ th column of the block $C_{i j}$. Obviously, $C_{i j}$ has at least one column (resp. two columns) if it corresponds to a real eigenvalue (resp. a complex eigenvalue pair). In addition, associated with each real eigenvalue $\lambda_{i}$ or complex eigenvalue pair $\left(\mu_{i}, \bar{\mu}_{i}\right)$, we define $C_{i}^{\ell}$ as the collection of the $\ell$ th columns of $C_{i j}$ (if they exist), namely,

$$
C_{i}^{\ell}=\left[\begin{array}{llll}
C_{i 1 \ell} & C_{i 2 \ell} & \ldots & C_{i r(i) \ell}
\end{array}\right] .
$$

The observability result presented below is a modification of [13], Theorem 5.21, for $A$ in the real Jordan form, where we recall that the pair $(C, A)$ is an observable pair if its corresponding unobservable subspace $\bar{O}(C, A)=\{0\}$.

Lemma 4.2. Let $A \in \mathbb{R}^{n \times n}$ and $C \in \mathbb{R}^{m \times n}$ with $A$ in the real Jordan form (4.1). Then $(C, A)$ is an observable pair if and only if the following two conditions are both valid:

(a) the columns of $C_{i}^{1}$ are linearly independent over the real field for all $i=1, \ldots, p$;

(b) the columns of both $C_{i}^{1}+\imath C_{i}^{2}$ and $C_{i}^{1}-\imath_{i} C_{i}^{2}$ are linearly independent over the complex field for all $i=p+1, \ldots, p+s$.

The next result shows that $C_{i j 1}$ plays a dominant role in characterizing the long time behavior associated with its real eigenvalue. 
Lemma 4.3. Let $J_{i j} \in \mathbb{R}^{k \times k}$ be a Jordan block defined above associated with a real eigenvalue $\lambda_{i}$ of $A$ and let its corresponding block in $C$ be $C_{i j}$ with $C_{i j 1} \neq 0$. Then for any nonzero $v \in \mathbb{R}^{k}$ with $v_{\ell}$ as the last nonzero element where $\ell \in\{1, \ldots, k\}, C_{i j} \mathrm{e}^{J_{i j} t} v=\mathrm{e}^{\lambda_{i} t}\left[\frac{t^{\ell-1}}{(\ell-1) !} C_{i j 1} v_{\ell}+O\left(t^{\ell-2}\right)\right]$.

Proof. Using the Jordan form of $J_{i j}$, we have

$$
\begin{aligned}
C_{i j} \mathrm{e}^{J_{i j} t} v & =C_{i j} \mathrm{e}^{t \lambda_{i}}\left[\begin{array}{ccccc}
1 & t & \frac{t^{2}}{2 !} & \cdots & \frac{t^{k-1}}{(k-1) !} \\
& 1 & t & \cdots & \frac{t^{k-2}}{(k-2) !} \\
& & \ddots & \ddots & \vdots \\
& & & 1 & t \\
& & & 1
\end{array}\right] v=\mathrm{e}^{\lambda_{i} t} C_{i j}\left[\begin{array}{c}
v_{\ell} \frac{t^{\ell-1}}{(\ell-1) !}+O\left(t^{\ell-2}\right) \\
O\left(t^{\ell-2}\right) \\
\vdots \\
O(1) \\
0 \\
\vdots \\
0
\end{array}\right] . \\
& =\mathrm{e}^{\lambda_{i} t}\left[\frac{t^{\ell-1}}{(\ell-1) !} C_{i j 1} v_{\ell}+O\left(t^{\ell-2}\right)\right]
\end{aligned}
$$

The following technical results, stated in a slightly general setting, are useful to characterize the longtime behaviors corresponding to complex eigenvalues of the matrix $A$. We call a continuous periodic function $h: \mathbb{R} \rightarrow \mathbb{R}$ with period $T>0$ of zero average if $\int_{0}^{T} h(\tau) \mathrm{d} \tau=0$.

Lemma 4.4. Given (finitely many) continuous periodic functions $g_{i}: \mathbb{R} \rightarrow\left[a_{i}, b_{i}\right]$ with frequency $\omega_{i}>0$, where $\left[a_{i}, b_{i}\right] \subseteq \mathbb{R}$ and $i=1, \ldots, m$. Assume that each $g_{i}$ is onto $\left[a_{i}, b_{i}\right]$ and the frequency ratio $\omega_{i} / \omega_{j}$ is irrational for any $i \neq j$. Then for any given $\widetilde{y} \in\left[a_{1}, b_{1}\right] \times \ldots \times\left[a_{m}, b_{m}\right]$ and any scalar $\varepsilon>0$, there is a $\tilde{t} \geq 0$ such that $\left\|\widetilde{y}-\left(g_{1}(\widetilde{t}), \ldots, g_{m}(\widetilde{t})\right)\right\|_{2} \leq \varepsilon$.

Proof. Let the function $g(t) \equiv\left(g_{1}(t), \ldots, g_{m}(t)\right)$, and let $\mathbb{T}^{m} \equiv \underbrace{\mathbb{S}^{1} \times \ldots \times \mathbb{S}^{1}}_{m \text {-times }}$ denote the $m$-torus. Moreover, let the functions $h: \mathbb{T}^{m} \rightarrow\left[a_{1}, b_{1}\right] \times \ldots \times\left[a_{m}, b_{m}\right]$ be $h\left(s_{1}, \ldots, s_{m}\right) \equiv\left(g_{1}\left(s_{1} / \omega_{1}\right), \ldots, g_{m}\left(s_{m} / \omega_{m}\right)\right)$ and $p: \mathbb{R} \rightarrow \mathbb{T}^{m}$ be $p(t) \equiv\left(\bmod \left(\omega_{1} t, 2 \pi\right), \ldots, \bmod \left(\omega_{m} t, 2 \pi\right)\right)$ respectively. It is easy to verify that $g=h \circ p$ and that $h$ is continuous and surjective. Due to the the irrational frequency ratios, the range of $p$ is dense on $\mathbb{T}^{m}[2]$, Example 3, p. 73. In particular, for any neighborhood $\mathcal{U}$ on $\mathbb{T}^{m}$, there exists $t^{\prime} \geq 0$ such that $p\left(t^{\prime}\right) \in \mathcal{U}$. For a given $\widetilde{y} \in\left[a_{1}, b_{1}\right] \times \ldots \times\left[a_{m}, b_{m}\right]$ and a given $\varepsilon>0$, it follows from the continuity and surjectivity of $h$ that $\mathcal{N} \equiv h^{-1}(\mathcal{B}(\widetilde{y}, \varepsilon))$ is a neighborhood on $\mathbb{T}^{m}$ of $h^{-1}(\widetilde{y})$, where $\mathcal{B}(\widetilde{y}, \varepsilon)$ denotes an open ball centered at $\widetilde{y}$ with the radius $\varepsilon$ (defined by the 2 -norm). We thus deduce, via the density of the range of $p$, that there is a $\widetilde{t} \geq 0$ such that $p(\widetilde{t}) \in \mathcal{N}$. Hence $\|\widetilde{y}-g(\widetilde{t})\|_{2} \leq \varepsilon$.

Lemma 4.5. Let $f_{i}: \mathbb{R} \rightarrow \mathbb{R}$ be a continuous and periodic function of zero average with frequency $\omega_{i}>0$, where $i=1, \ldots, m$ and $\omega_{i} \neq \omega_{j}$ for any $i \neq j$. Assume that $f(t):=\sum_{i=1}^{m} f_{i}(t)$ is not identically zero. Then there exist two scalars $\gamma_{1}>0$ and $\gamma_{2}<0$ such that for any $t_{*}, t_{1}, t_{2} \in\left[t_{*}, \infty\right)$ exist satisfying $f\left(t_{1}\right) \geq \gamma_{1}$ and $f\left(t_{2}\right) \leq \gamma_{2}$. 
Proof. Without loss of generality, we assume that each $f_{i}(t)$ is not identically zero. Since each $f_{i}$ is continuous, it achieves its maximal value $b_{i}$ and minimal value $a_{i}$ on any compact time interval of length $T_{i} \equiv 2 \pi / \omega_{i}$. Furthermore, since $f_{i}$ is not identically zero but has zero average, we must have $a_{i}<0<b_{i}$. For a given $t_{*}$, let $\widetilde{f}_{i}(s):=f_{i}\left(s+t_{*}\right)$, where $s:=t-t_{*}$. Note that $\widetilde{f}_{i}(s)$ is a continuous and periodic function satisfying all the properties of $f_{i}(t)$. Hence, without losing generality, we assume $t_{*}=0$. We consider three cases as follows:

(i) All the frequency ratios $\omega_{i} / \omega_{j}, i \neq j$ are rational. Hence, there exists a basis (i.e., resonant) frequency $\widetilde{\omega}>0$ such that for each $\omega_{i}, i=1, \ldots, m, \omega_{i}=n_{i} \widetilde{\omega}$ for some positive integer $n_{i}$. Thus $f(t)=\sum_{i=1}^{m} f_{i}(t)$ is continuous and periodic with frequency $\widetilde{\omega}(i . e$. , the period $T=2 \pi / \widetilde{\omega})$. Noticing that $T=n_{i} T_{i}$ such that $\int_{0}^{T} f_{i}(\tau) \mathrm{d} \tau=n_{i} \int_{0}^{T_{i}} f_{i}(\tau) \mathrm{d} \tau=0$, we obtain $\int_{0}^{T} f(\tau) \mathrm{d} \tau=\sum_{i=1}^{m} \int_{0}^{T} f_{i}(\tau) \mathrm{d} \tau=0$. Suppose $f(t) \geq 0($ resp. $\leq 0)$ on $[0, T]$. We deduce, via the continuity of $f$, that $f(t) \equiv 0$ on $[0, T]$. Therefore $f(t)$ is identically zero. This is a contradiction. Consequently, there must be two points $\widetilde{t}_{1}, \widetilde{t}_{2} \in[0, T]$ such that $f\left(\widetilde{t}_{1}\right) f\left(\widetilde{t}_{2}\right)<0$. Besides, since $f(t)$ is periodic, continuous and bounded, it must achieve its maximal and minimal values on any interval of length $T$, i.e., $t_{1}, t_{2} \in[0, T]$ exist such that $f\left(t_{1}\right)=\gamma_{1}$ and $f\left(t_{2}\right)=\gamma_{2}$, where $\gamma_{1}>0$ is the maximal value of $f$ and $\gamma_{2}<0$ is the minimal value of $f$.

(ii) All the frequency ratios $\omega_{i} / \omega_{j}$ with $i \neq j$ are irrational. Note that each function $f_{i}(t)$ is periodic and onto $\left[-a_{i}, b_{i}\right]$. Choose two points $\widetilde{y}_{1}=\left(b_{1}, \ldots, b_{m}\right)>0$ and $\widetilde{y}_{2}=\left(a_{1}, \ldots, a_{m}\right)<0$. By Lemma 4.4, we obtain $t_{1}, t_{2} \geq 0$ such that $f_{i}\left(t_{1}\right)$ is sufficiently close to $b_{i}$ and $f_{i}\left(t_{2}\right)$ is sufficiently close to $a_{i}$ for each $i$. Thus $f=\sum_{i=1}^{m} f_{i}$ is sufficiently close to $\sum_{i=1}^{m} b_{i}>0$ at $t_{1}$ and to $\sum_{i=1}^{m} a_{i}<0$ at $t_{2}$ respectively. The lemma follows by letting $\gamma_{1} \equiv \frac{1}{2} \sum_{i=1}^{m} b_{i}$ and $\gamma_{2} \equiv \frac{1}{2} \sum_{i=1}^{m} a_{i}$.

(iii) Some of $\omega_{i} / \omega_{j}$ with $i \neq j$ are rational and the others are irrational. Note that the rational ratio of a pair of frequencies defines an equivalent relation between the two frequencies. Therefore, for the family of the functions $f_{i}(t)$, we obtain a collection of (disjoint) equivalent classes $E_{\omega_{i}}=\left\{f_{j}(t) \mid \omega_{i} / \omega_{j}\right.$ is rational $\}$. Let $h_{E_{\omega_{i}}}(t):=\sum_{f_{j} \in E_{\omega_{i}}} f_{j}(t)$. Hence, $f(t)=\sum h_{E_{\omega_{i}}}(t)$. Moreover, at least one of the $h_{E_{\omega_{i}}}(t)$ 's is non-vanishing (i.e. it is not identically zero), since otherwise we have a contradiction that $f(t)$ is identically zero. Without loss of generality, we may assume that all the $h_{E_{\omega_{i}}}(t)$ 's are non-vanishing. It is easy to verify that each (nonvanishing) $h_{E_{\omega_{i}}}(t)$ is a continuous periodic function with zero average and the (basis) frequency $\widetilde{\omega}_{i}$. Thus it has maximal and minimal values $\mu_{i}>0$ and $\nu_{i}<0$ respectively. Moreover, for any two distinct equivalent classes, the ratio of their basis frequencies is irrational. Hence, by the similar argument as in (ii), we see that there exist $t_{1}, t_{2} \geq 0$ such that $f(t)$ is sufficiently close to $\sum \mu_{i}>0$ at $t_{1}$ and sufficiently close to $\sum \nu_{i}<0$ at $t_{2}$, respectively. This leads to the desired result.

Corollary 4.6. Let $f: \mathbb{R} \rightarrow \mathbb{R}$ be $f(t) \equiv \sum_{i=1}^{m}\left[\alpha_{i} \cos \left(\omega_{i} t\right)+\beta_{i} \sin \left(\omega_{i} t\right)\right]$, where $\omega_{i}>0, \omega_{i} \neq \omega_{j}$ for $i \neq j$, and $\left|\alpha_{i}\right|+\left|\beta_{i}\right| \neq 0$ for all $i$. Then there exist two scalars $\gamma_{1}>0$ and $\gamma_{2}<0$ such that for any $t_{*}$, two time instants $t_{1}, t_{2} \in\left[t_{*}, \infty\right)$ exist satisfying $f\left(t_{1}\right) \geq \gamma_{1}$ and $f\left(t_{2}\right) \leq \gamma_{2}$.

Proof. Let $f_{i}(t):=\alpha_{i} \cos \left(\omega_{i} t\right)+\beta_{i} \sin \left(\omega_{i} t\right)$. Apparently, each $f_{i}$ is a continuous periodic function with zero average and is not identically zero. We only need to verify that $f(t)=\sum_{i=1}^{m} f_{i}(t)$ is not identically zero. Notice that for any given $t_{*}$, we can write $f$ as $f\left(s+t_{*}\right)=\sum_{i=1}^{m}\left[\widetilde{\alpha}_{i} \cos \left(\omega_{i} s\right)+\widetilde{\beta}_{i} \sin \left(\omega_{i} s\right)\right]$, where $s:=t-t_{*}$, and

$$
\left[\begin{array}{c}
\widetilde{\alpha}_{i} \\
\widetilde{\beta}_{i}
\end{array}\right]=\left[\begin{array}{cc}
\cos \left(\omega_{i} t_{*}\right) & \sin \left(\omega_{i} t_{*}\right) \\
-\sin \left(\omega_{i} t_{*}\right) & \cos \left(\omega_{i} t_{*}\right)
\end{array}\right]\left[\begin{array}{c}
\alpha_{i} \\
\beta_{i}
\end{array}\right]
$$


Hence, $\left|\alpha_{i}\right|+\left|\beta_{i}\right| \neq 0$ if and only if $\left|\widetilde{\alpha}_{i}\right|+\left|\widetilde{\beta}_{i}\right| \neq 0$. Hence, without loss of generality, we consider $t_{*}=0$. Suppose that $f(t)$ is identically zero. Then by the Taylor expansion of $f(t)$ at $t_{*}=0$, we have

$$
\left[\begin{array}{cccc}
1 & 1 & \ldots & 1 \\
\omega_{1}^{2} & \omega_{2}^{2} & \ldots & \omega_{m}^{2} \\
\vdots & \vdots & & \vdots \\
\omega_{1}^{2(m-1)} & \omega_{2}^{2(m-1)} & \ldots & \omega_{m}^{2(m-1)}
\end{array}\right]\left[\begin{array}{c}
\alpha_{1} \\
\alpha_{2} \\
\vdots \\
\alpha_{m}
\end{array}\right]=0, \quad\left[\begin{array}{cccc}
1 & 1 & \ldots & 1 \\
\omega_{1}^{2} & \omega_{2}^{2} & \ldots & \omega_{m}^{2} \\
\vdots & \vdots & & \vdots \\
\omega_{1}^{2(m-1)} & \omega_{2}^{2(m-1)} & \ldots & \omega_{m}^{2(m-1)}
\end{array}\right]\left[\begin{array}{c}
\omega_{1} \beta_{1} \\
\omega_{2} \beta_{2} \\
\vdots \\
\omega_{m} \beta_{m}
\end{array}\right]=0 .
$$

Since the above matrix is a Vandermonde matrix with $\omega_{i}^{2} \neq \omega_{j}^{2}$ for all $i \neq j$, we have $\alpha_{i}=0$ and $\beta_{i}=0$, a contradiction. In fact, this can be extended to show that $f(t) \neq 0$ almost everywhere. Finally, by applying Lemma 4.5 , we obtain the desired result.

We write $\mathcal{X}_{C} \equiv\{x \mid C x \geq 0\}$ and let $E\left(\lambda_{i}\right)$ be the eigenspace associated with the real eigenvalue $\lambda_{i}$ of $A$. Moreover, we let $\mathcal{E}_{A}$ and $\mathcal{A}_{(C, A)}$ be the equilibrium set of $A$ and the attracting cone with respect to the pair $(C, A)$ respectively. Similarly, we let $\mathcal{E}_{(C, A)}:=\mathcal{E}_{A} \cap \mathcal{X}_{C}$ and $\mathcal{Y}_{(C, A)}$ denote the semiobservable cone associated with the pair $(C, A)$. Since $\mathcal{E}_{A}=E(0)$, it is easy to see that $\mathcal{E}_{(C, A)}=E(0) \cap \mathcal{X}_{C}$. We first give some necessary conditions in term of observability of each Jordan block and the corresponding block in $C$. These conditions are a direct consequence of basic linear systems theory, e.g., [13], and Lemma 4.2; the proof is thus omitted.

Lemma 4.7. Let $A \in \mathbb{R}^{n \times n}$ and $C \in \mathbb{R}^{m \times n}$ with $A$ in the real Jordan form (4.1) and $C$ in (4.2). Then the statement $\mathcal{E}_{(C, A)}=\mathcal{A}_{(C, A)}$ holds only if the following two conditions are both satisfied:

(a) for each real block $J_{i}$ (resp. Jordan block $J_{i j}$ ) associated with a nonzero real eigenvalue or a complex eigenvalue pair, $\left(C_{i}, J_{i}\right)$ (resp. $\left(C_{i j}, J_{i j}\right)$ ) is an observable pair;

(b) for each real block $J_{0}$ (resp. $J_{0 j}$ ) associated with the zero eigenvalue, $\bar{O}\left(C_{0}, J_{0}\right)=\operatorname{ker}\left[\begin{array}{c}C_{0} \\ J_{0}\end{array}\right]$ (resp. $\left.\bar{O}\left(C_{0 j}, J_{0 j}\right)=\operatorname{ker}\left[\begin{array}{c}C_{0 j} \\ J_{0 j}\end{array}\right]\right)$.

Moreover, for a real Jordan block $J_{i j}$ corresponding to a real eigenvalue (resp. a complex eigenvalue pair), the pair $\left(C_{i j}, J_{i j}\right)$ is observable if and only if $C_{i j 1} \neq 0$ (resp. $\left.\left[\begin{array}{ll}C_{i j 1} & C_{i j 2}\end{array}\right] \neq 0\right)$.

We introduce another notion. Let $F, G: \mathbb{R} \rightarrow \mathbb{R}^{\ell}$ be two functions. We say that $F(t)$ tends to $G(t)$ as $t \rightarrow+\infty$ if for any $\varepsilon>0$, there is $t_{\varepsilon} \geq 0$ such that $\|F(t)-G(t)\| \leq \varepsilon, \forall t \geq t_{\varepsilon}$. For ease of presentation, we present the characterizing conditions for $(C, A)$ in three cases.

Proposition 4.8. Consider the pair $(C, A)$ such that $\mathcal{E}_{(C, A)}=\{0\}$. Then $\mathcal{E}_{(C, A)}=\mathcal{A}_{(C, A)}$ if and only if the following conditions are both satisfied:

(a) statements (a) and (b) of Lemma 4.7 hold;

(b) $E\left(\lambda_{i}\right) \cap \mathcal{X}_{C}=\{0\}$ for each distinct nonzero real eigenvalue $\lambda_{i}$.

Moreover, (b) holds if and only if for each nonzero real eigenvalue $\lambda_{i}$, the linear inequality $C_{i}^{1} y \geq 0$ has the zero solution only, where $C_{i}^{1}$ is defined in (4.3) with $\ell=1$.

Proof. The "only if" part holds trivially; we only need to show the sufficiency. Without loss of generality, we assume that $A$ is in the real Jordan form. Notice that $\mathcal{E}_{(C, A)} \equiv E(0) \cap \mathcal{X}_{C}=\{0\}$ implies that $\operatorname{ker}\left[\begin{array}{c}C \\ A\end{array}\right]=\{0\}$, which further implies ker $\left[\begin{array}{c}C_{0} \\ J_{0}\end{array}\right]=\{0\}$. By (b) of Lemma 4.7, we have $\bar{O}\left(C_{0}, J_{0}\right)=\{0\}$, that is, $\left(C_{0}, J_{0}\right)$ is an observable pair. This result, along with (a) of Lemma 4.7, shows that $(C, A)$ is an observable pair and that 
each pair $\left(C_{i}, J_{i}\right)$ (resp. $\left.\left(C_{i j}, J_{i j}\right)\right)$ is observable for all $i, j$. Now consider an initial condition $v \in \mathcal{Y}_{(C, A)} \backslash \mathcal{E}_{(C, A)}$. Apparently, $v \neq 0$. Let $v^{J_{i j}}$ denote the sub-vector of $v$ corresponding to the $j$ th Jordan block of $J_{i}$. Hence, $C \mathrm{e}^{A t} v=\sum_{i, j} C_{i j} \mathrm{e}^{J_{i j} t} v^{J_{i j}}$. For presentation convenience, we look at three subcases as follows:

(i) $v^{J_{i j}}=0$ for all $i, j$ corresponding to the Jordan blocks associated with complex eigenvalues. In this case, the remaining sub-vectors correspond to the Jordan blocks associated with distinct real eigenvalues. Suppose, in contrast, that $C \mathrm{e}^{A t} v \geq 0, \forall t \geq 0$. Let $\lambda_{k}$ be the largest real eigenvalue such that $\left(v^{J_{k 1}}, \ldots, v^{J_{k r(k)}}\right) \neq 0$. Therefore, by observability of the pair $\left(C_{k}, J_{k}\right)$, we see that $C \mathrm{e}^{A t} v$ tends to $\sum_{j=1}^{r(k)} C_{k j} \mathrm{e}^{J_{k j} t} v^{J_{k j}}$ as $t \rightarrow+\infty$. Without loss of generality, we assume that $v^{J_{k j}} \neq 0$ for all $j=1, \ldots, r(k)$, since, otherwise we can simply drop those terms corresponding to the zero $v^{J_{k j}}$ in the subsequent analysis. For each sub-vector $v^{J_{k j}}$, let $v_{\ell_{j}}^{J_{k j}}$ be the last nonzero element of $v^{J_{k j}}$. Notice that Lemma 4.2 and the observability of the pair $\left(C_{k}, J_{k}\right)$ imply that $C_{k j 1} \neq 0$ for all $j$. By Lemma 4.3, we observe that $C_{k j} \mathrm{e}^{J_{k j} t} v^{J_{k j}}$ tends to $\mathrm{e}^{\lambda_{k} t} \frac{t^{\ell_{j}-1}}{\left(\ell_{j}-1\right) !} C_{k j 1} v_{\ell_{j}}^{J_{k j}}$ as $t \rightarrow+\infty$. We further assume, without losing generality, that $\ell_{1}=\ldots=\ell_{q}>\ell_{q+1}>\ldots>\ell_{r(k)}$ with $1 \leq q \leq r(k)$. Consequently, $\sum_{j=1}^{r(k)} C_{k j} \mathrm{e}^{J_{k j} t} v^{J_{k j}}$, and hence $C \mathrm{e}^{A t} v$, tends to

$$
\mathrm{e}^{\lambda_{k} t} \frac{t^{\ell_{1}-1}}{\left(\ell_{1}-1\right) !} \sum_{j=1}^{q} C_{k j 1} v_{\ell_{j}}^{J_{k j}} \equiv \mathrm{e}^{\lambda_{k} t} \frac{t^{\ell_{1}-1}}{\left(\ell_{1}-1\right) !} C_{k}^{1} y
$$

as $t \rightarrow+\infty$, where $y \equiv\left(v_{\ell_{1}}^{J_{k 1}}, \ldots, v_{\ell_{q}}^{J_{k q}}, 0, \ldots, 0\right)^{T}$ is nonzero. By augmenting $y$ to an $n$-vector $\widetilde{y}$, we obtain $0 \neq \widetilde{y} \in E\left(\lambda_{k}\right) \cap \mathcal{X}_{C}$, which is a contradiction to (b) of the proposition.

(ii) $v^{J_{i j}}=0$ for all $i, j$ corresponding to the Jordan blocks associated with real eigenvalues. Let $\mu_{k} \equiv \sigma_{k} \pm \imath \omega_{k}$ be the complex eigenvalues of $A$, where $k=1, \ldots, q$, such that the real parts of $\mu_{k}$ are the largest among those of all the complex eigenvalues of $A$ and that $\left(v^{J_{k 1}}, \ldots, v^{J_{k r(k)}}\right) \neq 0$. Note that we must have $\sigma_{1}=\ldots=\sigma_{q} \equiv \sigma$ and that $\omega_{i} \neq \omega_{k}$ for any $i, k \in\{1, \ldots, q\}$ with $i \neq k$. Since each pair $\left(C_{i j}, J_{i j}\right)$ is observable, $C_{i j} \mathrm{e}^{J_{i j} t} v^{J_{i j}}$ is not identically zero for any nonzero $v^{J_{i j}}$. Moreover, for each $J_{i j}$ of order $2 \ell$,

$$
\mathrm{e}^{J_{i j} t}=\left[\begin{array}{ccccc}
\mathrm{e}^{t D_{i}} & t \mathrm{e}^{t D_{i}} & \ldots & \ldots & \mathrm{e}^{t D_{i}} \frac{t^{\ell-1}}{(\ell-1) !} \\
& \mathrm{e}^{t D_{i}} & t \mathrm{e}^{t D_{i}} & \ldots & \mathrm{e}^{t D_{i}} \frac{t^{\ell-2}}{(\ell-2) !} \\
& & \ddots & \ddots & \vdots \\
& & & \ddots & t \mathrm{e}^{t D_{i}} \\
& & & & \mathrm{e}^{t D_{i}}
\end{array}\right], \quad \text { and } \quad \mathrm{e}^{D_{i} t}=\mathrm{e}^{\sigma t}\left[\begin{array}{cr}
\cos \left(\omega_{i} t\right) & \sin \left(\omega_{i} t\right) \\
-\sin \left(\omega_{i} t\right) & \cos \left(\omega_{i} t\right)
\end{array}\right]
$$

Hence, a straightforward computation shows that each element of $C_{i j} \mathrm{e}^{J_{i j} t} v^{J_{i j}}$ is of the form

$$
\mathrm{e}^{\sigma_{i} t} \sum_{l} \nu_{l} t^{l} \sin \left(\omega_{i} t+\theta_{l}\right)
$$

where $\nu_{l}$ and $\theta_{l}$ are constants depending on $v^{J_{i j}}$. Consequently, $C \mathrm{e}^{A t} v$ tends to $\sum_{k=1}^{q} \sum_{j=1}^{r(k)} C_{k j} \mathrm{e}^{J_{k j} t} v^{J_{k j}}$ as $t \rightarrow+\infty$. Specifically, at least one element of $C \mathrm{e}^{A t} v$ tends to $\mathrm{e}^{\sigma t} \sum_{k=1}^{q} \nu_{k} t^{\ell_{k}} \sin \left(\omega_{k} t+\theta_{k}\right)$ as $t \rightarrow+\infty$, where $\nu_{k}$ and $\theta_{k}$ are constants depending on $\left(v^{J_{k 1}}, \ldots, v^{J_{k r(k)}}\right)$. Without loss of generality, we assume $\ell_{1}=\ldots=\ell_{\bar{r}}>$ $\ell_{\bar{r}+1}>\ldots>\ell_{q}$ and $\nu_{k} \neq 0$ for all $1 \leq k \leq r$ with $1 \leq r \leq \bar{r}$. Hence, the element of $C \mathrm{e}^{A t} v$ considered above further tends to $g(t) \equiv \mathrm{e}^{\sigma t} t^{\ell_{1}} \sum_{k=1}^{r} \nu_{k} \sin \left(\omega_{k} t+\theta_{k}\right)$ as $t \rightarrow+\infty$. Since $\nu_{k} \neq 0$ and $\omega_{k}$ 's are distinct, we deduce 
the existence of $\gamma_{1}>0$ and $\gamma_{2}<0$, via Corollary 4.6, such that for any $t_{*} \geq 0$, there exist $t_{1}, t_{2} \in\left[t_{*}, \infty\right)$ with $g\left(t_{1}\right) \geq \gamma_{1} \mathrm{e}^{\sigma t_{1}}\left(t_{1}\right)^{\ell_{1}}>0$ and $g\left(t_{2}\right) \leq \gamma_{2} \mathrm{e}^{\sigma t_{2}}\left(t_{2}\right)^{\ell_{1}}<0$. This shows that $C \mathrm{e}^{A t_{*}} v \nsupseteq 0$ at some $t_{*}>0$.

(iii) We look at the case other than cases (i) and (ii). Let $v=\left(v_{r e}, v_{c p}\right)$, where $v_{r e} \neq 0$ and $v_{c p} \neq 0$ are the sub-vectors of $v$ corresponding to the real and complex eigenvalues respectively. Hence, $C \mathrm{e}^{A t} v=$ $C_{r e} \mathrm{e}^{J_{r e} t} v_{r e}+C_{c p} \mathrm{e}^{J_{c p} t} v_{c p}$, where $J_{r e}$ and $J_{c p}$ are the respective Jordan blocks associated with the real and complex eigenvalues. Following the result of (i), we see that one element of $C_{r e} \mathrm{e}^{J_{r e} t} v_{r e}$ tends to $\mathrm{e}^{\bar{\lambda} t} t^{\bar{\ell}} \alpha$ with $\alpha<0$ as $t \rightarrow+\infty$. For the same corresponding element of $C_{c p} \mathrm{e}^{J_{c p} t} v_{c p}$, either (1) such the element is identically zero for all $t$, or (2) such the element is not identically zero. For the former case, it is obvious that the sufficiency holds. For the latter case, we have, via the similar argument as (ii), that this element tends to $\widetilde{g}(t) \equiv \mathrm{e}^{\widetilde{\sigma} t} t^{\widetilde{\ell}} \sum_{k=1}^{\widetilde{r}} \widetilde{\nu}_{k} \sin \left(\widetilde{\omega}_{k} t+\theta_{k}\right)$ as $t \rightarrow+\infty$, where $\widetilde{\nu}_{k} \neq 0$ and $\widetilde{\omega}_{k}$ 's are distinct. Consider two additional sub-cases:

(2.1) $(\bar{\lambda}, \bar{\ell}) \neq(\widetilde{\sigma}, \widetilde{\ell})$. In this case, either $\mathrm{e}^{\bar{\lambda} t} t^{\bar{\ell}} \alpha$ or $\widetilde{g}(t)$ dominates for all $t \geq 0$ sufficiently large. Hence, the argument of (i) or (ii) leads to the sufficiency.

$(2.2)(\bar{\lambda}, \bar{\ell})=(\widetilde{\sigma}, \widetilde{\ell})$. In this case, the corresponding element of $C \mathrm{e}^{A t} v$ tends to $h(t) \equiv \mathrm{e}^{\tilde{\sigma} t} t^{\tilde{\ell}}[\alpha+$ $\left.\sum_{k=1}^{\widetilde{r}} \widetilde{\nu}_{k} \sin \left(\widetilde{\omega}_{k} t+\theta_{k}\right)\right]$ as $t \rightarrow+\infty$, where $\alpha<0$. Moreover, we deduce, via Corollary 4.6, the existence of $\gamma_{2}<0$ such that for any $\widetilde{t} \geq 0$, there is $t^{\prime} \geq \widetilde{t}$ satisfying $\sum_{k=1}^{\widetilde{r}} \widetilde{\nu}_{k} \sin \left(\widetilde{\omega}_{k} t^{\prime}+\theta_{k}\right) \leq \gamma_{2}<0$, which further shows that $h\left(t^{\prime}\right)<\mathrm{e}^{\bar{\lambda} t^{\prime}}\left(t^{\prime}\right)^{\bar{\ell}}\left(\alpha+\gamma_{2}\right)<0$. This results in the sufficiency.

Lastly, we show the equivalent condition for (b). For each distinct real eigenvalue $\lambda_{i}$, we see from the real Jordan form of $A$ that the eigenspace $E\left(\lambda_{i}\right)=\operatorname{Span}\left\{\mathbf{e}_{i j 1}\right\}_{j=1}^{r(i)}$, where $\mathbf{e}_{i j 1}$ is the standard basis vector whose nonzero element corresponds to the first column of each Jordan block $J_{i j}$ associated with $\lambda_{i}$. By the given form of $C$, we thus deduce that $E\left(\lambda_{i}\right) \cap \mathcal{X}_{C}=\{0\}$ if and only if $\left\{y \mid \sum_{j=1}^{r(i)} C_{i j 1} y_{j} \geq 0\right\}=\left\{y \mid C_{i}^{1} y \geq 0\right\}$ contains the zero element only. This leads to the desired result.

Remark 4.9. It is worth pointing out that Proposition 4.8 can be proved by Theorem 3.2. Indeed, the condition $\mathcal{E}_{(C, A)}=\{0\}$ implies that $\mathcal{E}_{(C, A)}=\mathcal{A}_{(C, A)}$ is equivalent to $\mathcal{A}_{(C, A)}=\{0\}$. Hence, the observability condition (a) and the eigenspace condition (b) of Proposition 4.8 can be obtained by applying Theorem 3.2. Admittedly, Theorem 3.2 is based upon an elegant fixed point argument that gives rise to a shorter proof, while Proposition 4.8, on the other hand, relies on the dynamical system results, e.g., Lemmas 4.4 and 4.5, which lead to a longer, yet more constructive, alternative proof. Nevertheless, the latter arguments form a foundation for the investigation of other cases of CLSs with infinite mode switchings as seen below.

To avoid redundancy in the subsequent development, we always assume that $A$ is of the real Jordan form. Furthermore, recall that $J_{0}$ is the Jordan block of $A$ associated with the zero eigenvalue and $C_{0}$ is its corresponding matrix block in $C$.

Proposition 4.10. Consider the pair $(C, A)$ such that $\mathcal{E}_{(C, A)} \cap \operatorname{int} \mathcal{X}_{C} \neq \emptyset$. Then $\mathcal{E}_{(C, A)}=\mathcal{A}_{(C, A)}$ holds if and only if the following four conditions are all satisfied:

(a) statements (a) and (b) of Lemma 4.7 hold;

(b) each nonzero real eigenvalue $\lambda_{i}$ of $A$ is positive and $E\left(\lambda_{i}\right) \cap \mathcal{X}_{C}=\{0\}$;

(c) each complex eigenvalue of $A$ has the positive real part;

(d) $\left[C_{0} \mathrm{e}^{J_{0} t} v_{0} \geq 0, \forall t \geq 0\right] \Longrightarrow J_{0} v_{0}=0$.

Moreover, (d) holds only if $J_{0 j}$ is a scalar, i.e., $J_{0 j}=0$, for any $j \in\left\{1, \ldots, r_{0}\right\}$ such that $C_{0 j 1} \geq 0$ or $C_{0 j 1} \leq 0$, where $J_{0 j}$ is the Jordan block associated with the zero eigenvalue. 
Proof. "Necessity". We only need to show (b)-(d) as follows:

(1.1) Consider (b) first, for which we only need to show $\lambda_{i}>0$ if $\lambda_{i} \neq 0$. Suppose, in contrast, that $\lambda_{i}<0$. Let $u \neq 0$ be an eigenvector of $A$ associated with $\lambda_{i}$. Since $\mathcal{E}_{A} \cap \operatorname{int} \mathcal{X}_{C} \neq \emptyset$, a nonzero vector $v^{0}$ exists such that $A v^{0}=0$ and $C v^{0}>0$. Hence, for any real $\varepsilon>0, v^{0}+\varepsilon u$ is not an equilibrium and $C \mathrm{e}^{A t}\left[v^{0}+\varepsilon u\right]=C v^{0}+\mathrm{e}^{\lambda_{i} t} \varepsilon C u$. Since $\mathrm{e}^{\lambda_{i} t} \leq 1, \forall t \geq 0$, we have $C v^{0}+\mathrm{e}^{\lambda_{i} t} \varepsilon C u>0, \forall t \geq 0$ for all $\varepsilon>0$ sufficiently small. This shows that $\mathrm{e}^{A t}\left[v^{0}+\varepsilon u\right] \in \mathcal{X}_{C}, \forall t \geq 0$ for all $\varepsilon>0$ small, which is a contradiction.

(1.2) The statement (c) can be proved via the similar argument as (1.1).

(1.3) Consider (d). Suppose, in contrast, that there exists a vector $v_{0}$ such that $J_{0} v_{0} \neq 0$ and $C_{0} \mathrm{e}^{J_{0} t} v_{0} \geq 0$, $\forall t \geq 0$. Since $J_{0}$ has the zero eigenvalue only, we see that $v_{0}$ does not belong to the eigenspace of $J_{0}$ (associated with the zero eigenvalue) and thus the vector $x^{0}=\left(v_{0}, 0\right)$ is not an equilibrium in $\mathbb{R}^{n}$. Moreover, $C \mathrm{e}^{A t} x^{0}=$ $C_{0} \mathrm{e}^{J_{0} t} v_{0} \geq 0, \forall t \geq 0$ by the assumption. Thus $\mathrm{e}^{A t} x^{0} \in \mathcal{X}_{C}, \forall t \geq 0$. This results in a contradiction.

(1.4) The necessary condition for (d). Suppose, in contrast, that a Jordan block $J_{0 j}$ is of order 2 and its corresponding block $C_{0 j 1} \geq 0$ or $C_{0 j 1} \leq 0$. Consider the case where $C_{0 j 1} \geq 0$ first. Let $\mathbf{e}_{0 j 2}$ be the standard basis vector of $\mathbb{R}^{n}$ whose nonzero element corresponds to the second column of $J_{0 j}$. Moreover, as in (1.1), let $v^{0}$ be such that $A v^{0}=0$ and $C v^{0}>0$. Let an initial state be $v^{0}+\varepsilon \mathbf{e}_{0 j 2}$ with $\varepsilon>0$. We deduce, from the construction, that $v^{0}+\varepsilon \mathbf{e}_{0 j 2} \notin E(0)$ and $C \mathrm{e}^{A t}\left[v^{0}+\varepsilon \mathbf{e}_{0 j 2}\right]=C v^{0}+\varepsilon C_{j 02}+t \varepsilon C_{j 01}$. Hence, $v^{0}+\varepsilon \mathbf{e}_{0 j 2}$ is not an equilibrium and $C \mathrm{e}^{A t}\left[v^{0}+\varepsilon \mathbf{e}_{0 j 2}\right]>0, \forall t \geq 0$ for all $\varepsilon>0$ sufficiently small. The case where $C_{0 j 1} \leq 0$ can be proved in the similar way.

"Sufficiency". We prove it by contradiction. Suppose that a vector $x^{0}$ exists such that $x^{0} \notin \mathcal{E}_{(C, A)}$ but $v \in \mathcal{A}_{(C, A)}$. Let $x^{0}=\left(v_{0}, v_{r e \overline{0}}, v_{c p}\right)$, where the three sub-vectors $v_{0}, v_{r e \overline{0}}, v_{c p}$ correspond to the zero eigenvalue, the nonzero real eigenvalues, and the complex eigenvalues respectively. If $\left(v_{r e \overline{0}}, v_{c p}\right) \neq 0$, then by the observability condition in (a), the eigenvalue conditions in (b) and (c) (i.e., $\lambda_{i}>0$ if $\lambda_{i} \neq 0$ and $\sigma_{j}>0$ for any complex eigenvalues $\sigma_{j} \pm \omega_{j}$ ), and the similar argument as (i)-(iii) of Proposition 4.8, we conclude that a $t_{*}>0$ exists such that $C \mathrm{e}^{A t_{*}} v \nsupseteq 0$, which is a contradiction to $x^{0} \in \mathcal{A}_{(C, A)}$. Therefore, $\left(v_{r e \overline{0}}, v_{c p}\right) \equiv 0$ or equivalently $x^{0}=\left(v_{0}, 0,0\right)$. Since $x^{0} \notin \mathcal{E}_{A}, v_{0}$ is not in the null space of $J_{0}$ or equivalently the eigenspace of $J_{0}$. On the other hand, $x^{0} \in \mathcal{A}_{(C, A)}$ implies that $C_{0} \mathrm{e}^{J_{0} t} v^{J_{0}} \geq 0$ for all $t \geq 0$. This leads to a contradiction to $(\mathrm{d})$.

Proposition 4.11. Consider the pair $(C, A)$ such that $\mathcal{E}_{A} \cap \operatorname{int} \mathcal{X}_{C}=\emptyset$ but $\mathcal{E}_{A} \cap$ bd $\mathcal{X}_{C} \neq\{0\}$. Then $\mathcal{E}_{(C, A)}=\mathcal{A}_{(C, A)}$ holds only if the following conditions are all satisfied:

(a) statements (a) and (b) of Lemma 4.7 hold;

(b) $E\left(\lambda_{i}\right) \cap \mathcal{X}_{C}=\{0\}$ for each nonzero real eigenvalue $\lambda_{i}$;

(c) for any nonzero vector $v^{0} \in \mathcal{E}_{A} \cap \operatorname{bd} \mathcal{X}_{C}$ with $\left(C v^{0}\right)_{\alpha}>0$ and $\left(C v^{0}\right)_{\bar{\alpha}}=0$ (where $\bar{\alpha} \neq \emptyset$ ), the following two conditions both hold:

(c1) $E\left(\lambda_{i}\right) \cap \mathcal{X}_{C_{\bar{\alpha}}}=\{0\}$ for each negative real eigenvalue $\lambda_{i}$;

(c2) $\left(\left(C_{i}\right)_{\bar{\alpha} \bullet}, J_{i}\right)$ is an observable pair for each real block $J_{i}$ associated with a complex eigenvalue pair $\sigma_{i} \pm \imath \omega_{i}$ with $\sigma_{i} \leq 0$

(d) $\left[C_{0} \mathrm{e}^{J_{0} t} v_{0} \geq 0, \forall t \geq 0\right] \Longrightarrow J_{0} v_{0}=0$.

In addition, if (a)-(c) hold and $J_{0}=0$ (i.e., $J_{0}$ is diagonal), then $\mathcal{E}_{(C, A)}=\mathcal{A}_{(C, A)}$.

Proof. "Necessity". We only need to show (c) since (a), (b) and (d) have been shown before.

(c1) Suppose, in contrast, that there exist a nonzero vector $v^{0} \in \mathcal{E}_{A} \cap$ bd $\mathcal{X}_{C}$ with $\left(C v^{0}\right)_{\alpha}>0$ and $\left(C v^{0}\right)_{\bar{\alpha}}=0$ and a nonzero vector $u \in E\left(\lambda_{i}\right) \cap \mathcal{X}_{C_{\bar{\alpha}}}$ for an eigenvalue $\lambda_{i}<0$. Thus $\left(C \mathrm{e}^{A t} u\right)_{\bar{\alpha}}=\mathrm{e}^{\lambda_{i} t}(C u)_{\bar{\alpha}} \geq 0$. A similar 
argument as (1.1) of Proposition 4.10 shows that $C_{\alpha} \bullet \mathrm{e}^{A t}\left[v^{0}+\varepsilon u\right]>0, \forall t \geq 0$ for all $\varepsilon>0$ small. Hence $C \mathrm{e}^{A t}\left[v^{0}+\varepsilon u\right]=\left[\begin{array}{c}C_{\alpha} \mathrm{e}^{A t}\left[v^{0}+\varepsilon u\right] \\ \varepsilon \mathrm{e}^{\lambda_{i} t}(C u)_{\bar{\alpha}}\end{array}\right] \geq 0, \forall t \geq 0$ for all $\varepsilon>0$ small. Since $v^{0}+\varepsilon u$ is not an equilibrium, we have a contradiction.

(c2) Suppose, in contrast, that there exist a $0 \neq v^{0} \in \mathcal{E}_{A} \cap$ bd $\mathcal{X}_{C}$ with $\left(C v^{0}\right)_{\alpha}>0$ and $\left(C v^{0}\right)_{\bar{\alpha}}=0$ and a complex eigenvalue pair $\sigma_{i} \pm \imath \omega_{i}$ with $\sigma_{i} \leq 0$ such that $\left(\left(C_{i}\right)_{\bar{\alpha} \bullet}, J_{i}\right)$ is an unobservable pair. Hence, by basic linear systems theory, we obtain a (nonzero) vector $u \in \operatorname{Span}\left\{\mathbf{e}_{i 11}, \mathbf{e}_{i 12}, \ldots, \mathbf{e}_{i r(i) 1}, \mathbf{e}_{i r(i) 2}\right\}$ with real weights $\left(c_{11}, c_{12}, \ldots, c_{r(i) 1}, c_{r(i) 2}\right) \neq 0$ such that $C_{\bar{\alpha} \bullet} \mathrm{e}^{A t} u=0, \forall t \geq 0$, where

$$
C \mathrm{e}^{A t} u=\sum_{j=1}^{r(i)}\left[\begin{array}{ll}
C_{i j 1} & C_{i j 2}
\end{array}\right] \mathrm{e}^{D_{i} t}\left(\begin{array}{c}
c_{j 1} \\
c_{j 2}
\end{array}\right), \quad \text { and } \quad D_{i}=\left[\begin{array}{cc}
\sigma_{i} & \omega_{i} \\
-\omega_{i} & \sigma_{i}
\end{array}\right]
$$

Furthermore, $v^{0}+\varepsilon u$ is not an equilibrium for $\varepsilon>0$ sufficiently small and $C \mathrm{e}^{A t}\left[v^{0}+\varepsilon u\right]=C v^{0}+\varepsilon C \mathrm{e}^{A t} u$. Since $\sigma_{i} \leq 0,\left\|\mathrm{e}^{D_{i} t}\right\|$ is bounded for all $t \geq 0$. Therefore, $C_{\alpha} \bullet \mathrm{e}^{A t} u$ is bounded for all $t \geq 0$. This, together with the fact that $\left(C v^{0}+C \mathrm{e}^{A t} u\right)_{\bar{\alpha} \bullet} \equiv 0$ for all $t \geq 0$, shows that $C \mathrm{e}^{A t}\left[v^{0}+\varepsilon u\right] \geq 0, \forall t \geq 0$ for all $\varepsilon>0$ sufficient small. This is a contradiction.

We now prove the stated sufficient condition. Suppose that a vector $v$ exists such that $v \notin \mathcal{E}_{(C, A)}$ but $v \in \mathcal{A}_{(C, A)}$. Let $v=\left(v_{0}, v_{r e+}, v_{r e-}, v_{c p+}, v_{c p \leq 0}\right)$, where the sub-vectors $v_{0}, v_{r e+}, v_{r e-}, v_{c p+}, v_{c p \leq 0}$ correspond to the zero eigenvalue, the positive real eigenvalues, the negative real eigenvalues, the complex eigenvalues with positive real parts, and the complex eigenvalues with nonpositive real parts respectively. Since $J_{0}=0, v \notin \mathcal{E}_{A}$ if and only if $\left(v_{r e+}, v_{r e-}, v_{c p+}, v_{c p \leq 0}\right) \neq 0$. Note that if $\left(v_{r e+}, v_{c p+}\right) \neq 0$, then by the observability condition in (a) and the similar argument of Proposition 4.8, we conclude that a $t_{*}>0$ exists such that $C \mathrm{e}^{A t_{*}} v \geq 0$, which is a contradiction to $v \in \mathcal{A}_{(C, A)}$. Therefore, $\left(v_{r e+}, v_{c p+}\right) \equiv 0$ or equivalently $v=\left(v_{0}, 0, v_{r e-}, 0, v_{c p \leq 0}\right)$ with $\left(v_{r e-}, v_{c p \leq 0}\right) \neq 0$. We consider two cases as follows:

(i) $v_{0}=0$. In this case, $C \mathrm{e}^{A t} v=C_{r e-} \mathrm{e}^{J_{r e-} t} v_{r e-}+C_{c p \leq 0} \mathrm{e}^{J_{c p \leq 0} t} v_{c p \leq 0}$, where $J_{r e-}, J_{c p \leq 0}$ are the corresponding blocks in $A$ and $C_{r e-}, C_{c p \leq 0}$ are the corresponding blocks in $C$ respectively. Therefore the observability condition in (a), the condition (b), and the similar argument in Proposition 4.8 yields a $t_{*}>0$ such that $C \mathrm{e}^{A t_{*}} v \nsupseteq 0$, which is a contradiction.

(ii) $v_{0} \neq 0$. In this case, $C_{0} v_{0} \geq 0$ such that $v^{0}=\left(v_{0}, 0,0,0,0\right) \in \mathcal{E}_{A} \cap \mathcal{X}_{C}$ and thus the nonzero vector $v^{0} \in \mathcal{E}_{A} \cap \operatorname{bd} \mathcal{X}_{C}$ satisfies $\left(C v^{0}\right)_{\alpha}=\left(C_{0} v_{0}\right)_{\alpha}>0$ and $\left(C v^{0}\right)_{\bar{\alpha}}=\left(C_{0} v_{0}\right)_{\bar{\alpha}}=0$, where the index subset $\bar{\alpha}$ is nonempty. Hence,

$$
\begin{aligned}
C_{\bar{\alpha} \bullet} \mathrm{e}^{A t} v & =\left(C_{0} v_{0}\right)_{\bar{\alpha}}+\left(C_{r e-}\right)_{\bar{\alpha} \bullet} \mathrm{e}^{J_{r e-} t} v_{r e-}+\left(C_{c p \leq 0}\right)_{\bar{\alpha} \bullet} \mathrm{e}^{J_{c p} \leq 0}{ }^{t} v_{c p \leq 0} \\
& =\left(C_{r e-}\right)_{\bar{\alpha} \bullet} \mathrm{e}^{J_{r e-} t} v_{r e-}+\left(C_{c p \leq 0}\right)_{\bar{\alpha} \bullet} \mathrm{e}^{J_{c p \leq 0} t} v_{c p \leq 0} .
\end{aligned}
$$

By the equivalent condition for (b) of Proposition 4.8, we notice that $E\left(\lambda_{i}\right) \cap \mathcal{X}_{C_{\bar{\alpha}}}=\{0\}$ if and only if

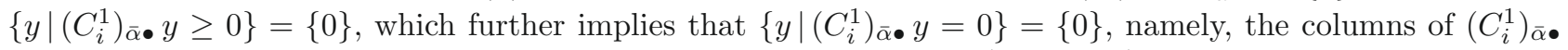
are linearly independent. We thus deduce via (a) of Lemma 4.2 that $\left(\left(C_{i}\right)_{\bar{\alpha} \bullet}, J_{i}\right)$ is an observable pair for each negative real eigenvalue $\lambda_{i}$. This observation, together with $\left(v_{r e-}, v_{c p \leq 0}\right) \neq 0$ and the similar argument in Proposition 4.8 , yields a $t_{*}>0$ such that $C_{\bar{\alpha}} \bullet \mathrm{e}^{A t_{*}} v \nsupseteq 0$, which is a contradiction.

\subsubsection{Finite verification}

The characterization results of Propositions 4.8,4.10, and 4.11 raise the question of whether the conditions in these propositions can be verified by a finite procedure, namely, whether checking these conditions is a decidable problem. It is easy to see that the conditions (a)-(c) in each proposition can be formulated as linear equations or linear inequalities, which can be checked via efficient linear programming methods in finite steps. 
Proposition 4.13 below addresses the condition (d) using the following sum-of-squares lemma in elementary algebra [30]:

Lemma 4.12. Let $f: \mathbb{R} \rightarrow \mathbb{R}$ be a (univariate) polynomial. Then $f(t) \geq 0, \forall t \in \mathbb{R}$ if and only if $f(t)=\sum_{i=1}^{k} g_{i}^{2}(t)$ for finitely many polynomials $g_{1}, \ldots, g_{k}$.

Proposition 4.13. Checking the condition (d) in Propositions 4.10 and 4.11 is a decidable problem.

Proof. Since $J_{0}$ has the zero eigenvalue only, it is nilpotent, i.e., $\left(J_{0}\right)^{\ell}=0$ for some positive integer $\ell$. Therefore $C_{0} \mathrm{e}^{J_{0} t} v_{0}=\sum_{k=1}^{\ell-1} \frac{C_{0}\left(J_{0}\right)^{k} v_{0}}{k !} t^{k}$ is a (vector-valued) polynomial in $t$ whose coefficients are linear combinations of $v_{0} \in \mathbb{R}^{n_{0}}$. Letting $t=s^{2}$ where $s \in \mathbb{R}$, we have $F\left(s, v_{0}\right) \equiv \sum_{k=1}^{\ell-1} \frac{C_{0}\left(J_{0}\right)^{k} v_{0}}{k !} s^{2 k}$ as a (vector-valued) polynomial in $\left(s, v_{0}\right) \in \mathbb{R}^{1+n_{0}}$. Moreover, the condition $J_{0} v_{0} \neq 0$ is equivalent to $h\left(v_{0}\right):=\left(J_{0} v_{0}\right)^{T} J_{0} v_{0}>0$, where $h\left(v_{0}\right)$ is a quadratic polynomial. Hence, checking the condition (d) is equivalent to the following problem whose defining functions are all polynomials:

$$
\left(\widetilde{\mathrm{P}}_{0}\right): \text { find } v_{0} \in \mathbb{R}^{n_{0}} \text { such that } F\left(s, v_{0}\right) \geq 0, \forall s \in \mathbb{R} \text { and } h\left(v_{0}\right)>0 .
$$

Let $F_{j}$ be the $j$ th element of $F\left(s, v_{0}\right), j=1, \ldots, m$. Hence, $F_{j}\left(s, v_{0}\right)=\left(a_{j 0}^{T} v_{0}\right)+\left(a_{j 1}^{T} v_{0}\right) s^{2}+\ldots+\left(a_{j k_{j}}^{T} v_{0}\right) s^{2 k_{j}}$, where the vectors $a_{j i}$ depend on $C_{0}$ and $J_{0}$ only. By Lemma $4.12, F_{j}\left(s, v_{0}\right) \geq 0, \forall s \in \mathbb{R}$ for some given $v_{0}$ if and only if $F_{j}\left(s, v_{0}\right)=z^{T} Q_{j}\left(v_{0}\right) z$, where $z=\left(1, s, \ldots, s^{k_{j}}\right)^{T}$ and $Q_{j}\left(v_{0}\right)$ is a symmetric and positive semidefinite matrix. Since the latter condition is equivalent to nonnegativity of all the principal minors of $Q_{j}\left(v_{0}\right)$ and each principal minor is a polynomial of the elements of $Q_{j}\left(v_{0}\right)$, the condition $F_{j}\left(s, v_{0}\right) \geq 0, \forall s \in \mathbb{R}$ holds for some $v_{0}$ if and only if finitely many (real-valued) polynomial inequalities $g_{j \ell}\left(q_{j}\right) \geq 0$ and finitely many (real-valued) polynomial (indeed, linear) equations $l_{j i}\left(q_{j}, v_{0}\right):=b_{j i}^{T} q_{j}-a_{j i}^{T} v_{0}=0, i=0, \ldots, k_{j}$ have a real solution $\left(\widetilde{q}_{j}, \widetilde{v}_{0}\right)$, where $q_{j} \in \mathbb{R}^{\left(2+k_{j}\right)\left(1+k_{j}\right) / 2}$ corresponds to the independent elements in $Q_{j}, b_{j i} \in \mathbb{R}^{\left(2+k_{j}\right)\left(1+k_{j}\right) / 2}$ are constant vectors, and the polynomial inequalities $g_{i \ell}\left(q_{j}\right) \geq 0$ and the polynomial equations $l_{j i}\left(q_{j}, v_{0}\right)=0$ correspond to the principal minor conditions and the coefficient constraints respectively. Letting $q=\left(q_{1}, \ldots, q_{m}\right) \in \mathbb{R}^{n_{q}}$ and observing that feasibility of $\left(\widetilde{\mathrm{P}}_{0}\right)$ is equivalent to existence of $v_{0}$ satisfying $F_{j}\left(s, v_{0}\right) \geq 0, \forall s \in \mathbb{R}$ for all $j=1, \ldots, m$ and $h\left(v_{0}\right)>0$, we deduce that the problem $\left(\widetilde{\mathrm{P}}_{0}\right)$ is equivalent to the subsequent semi-algebraic problem:

$$
\left(\widehat{\mathrm{P}}_{0}\right): \text { find }\left(q, v_{0}\right) \in \mathbb{R}^{n_{q}+n_{0}} \quad \text { such that } g_{j \ell}(q) \geq 0, \quad l_{j i}\left(q, v_{0}\right)=0, \forall j, \ell, i, \text { and } h\left(v_{0}\right)>0 \text {. }
$$

Following Seidenberg's approach in real algebraic geometry, e.g., [7], Fact 2.5, one can show that verifying (in)solvability of the problem $\left(\widehat{\mathrm{P}}_{0}\right)$ is equivalent to checking whether finitely many multivariate polynomial equations have real solutions. The latter problem can be solved by the Tarski-Seidenberg decision procedure in finite steps $[6,7]$. Consequently verification of the original condition (d) is decidable.

Proposition 4.13 not only shows decidability of checking the condition (d) but its proof also leads to an algorithm to verify $(\mathrm{d})$. However, solving a semi-algebraic problem such as $\left(\widehat{\mathrm{P}}_{0}\right)$ via the decision procedure is known to be algorithmically hard (in term of complexity), and it is expected that a more efficient algorithm can be developed by exploiting the problem structure. Nevertheless, further exploration of this issue is beyond the scope of the paper. We refer the reader to $[3,6,7,30]$ for additional discussion on the algorithmic issues and related mathematical programming approaches respectively. 


\subsection{Bimodal CLSs}

Bimodal CLSs are the simplest, yet nontrivial, CLSs with two modes. It has been shown in [11], Example 2.1, that each cone of such a CLS is a half space of $\mathbb{R}^{n}$ and the bimodal CLS can be written as $\dot{x}=A x+b \max \left(0,-c^{T} x\right)$ for some $n \times n$ matrix $A$ and two (nonzero) $n$-vectors $b, c$. In this section, we derive necessary and sufficient conditions that characterize bimodal CLSs with infinite mode switchings. As discussed in the previous section, we can focus on the mode corresponding to the pair $\left(c^{T}, A\right)$. An important feature of the cone $\mathcal{X}_{c^{T}} \equiv\left\{x \mid c^{T} x \geq 0\right\}$ is that it is non-pointed so that it has a nontrivial intersection with any nonzero subspace. In the following, we assume that $A$ is in the real Jordan form and let $c_{J_{i}}^{T}$ be the sub-row in $c^{T}$ corresponding to the block $J_{i}$ of $A$. Moreover, we let $J_{0}$ be the block associated with the zero eigenvalue and $c_{J_{0}}^{T}$ be the corresponding sub-row in $c^{T}$.

Theorem 4.14. $\mathcal{E}_{\left(c^{T}, A\right)}=\mathcal{A}_{\left(c^{T}, A\right)}$ if and only if the following conditions are all satisfied:

(a) if $\mathcal{E}_{A} \neq\{0\}$, then the matrix block $J_{0}=0$ (i.e., $J_{0}$ is diagonal);

(b) A has no nonzero real eigenvalue;

(c) for each real block $J_{i}$ associated with a complex eigenvalue pair, $\left(c_{J_{i}}^{T}, J_{i}\right)$ is an observable pair;

(d) if $\mathcal{E}_{A} \neq\{0\}$ and $c_{J_{0}}^{T} \neq 0$, then each complex eigenvalue of $A$ has the positive real part.

Moreover, (c) holds if and only if $J_{i}$ has a single Jordan block and $\left(c_{i 1}, c_{i 2}\right) \neq 0$.

Proof. "Necessity". We consider (a) first. Suppose that $J_{0}$ is not diagonal. Then there is a real Jordan block $J_{0 j}$ associated with the zero eigenvalue such that $J_{0 j}$ is of order 2 . Consider the initial state $v^{0}=\alpha_{1} \mathbf{e}_{0 j 1}+\alpha_{2} \mathbf{e}_{0 j 2}$ for $\alpha_{1}, \alpha_{2} \in \mathbb{R}$. A straightforward computation gives rise to $c^{T} \mathrm{e}^{A t} v^{0}=\left(\alpha_{1} c_{0 j 1}+\alpha_{2} c_{0 j 2}\right)+\alpha_{2} c_{0 j 1} t$. Consider three cases as follows: (a.1) $c_{0 j 1} \neq 0$. Letting $\alpha_{1}=\operatorname{sgn}\left(c_{0 j 1}\right)$ and $\alpha_{2}=\varepsilon \operatorname{sgn}\left(c_{0 j 1}\right)$ where $\varepsilon>0$, we have $c^{T} \mathrm{e}^{A t} v^{0}=\operatorname{sgn}\left(c_{0 j 1}\right)\left[\left(c_{0 j 1}+\varepsilon c_{0 j 2}\right)+c_{0 j 1} t\right]>0, \forall t \geq 0$ for all $\varepsilon>0$ sufficiently small; $($ a.2 $) c_{0 j 1}=0$ but $c_{0 j 2} \neq 0$. Letting $\alpha_{1}$ be arbitrary and $\alpha_{2}=\operatorname{sgn}\left(c_{0 j 2}\right)$, we have $c^{T} \mathrm{e}^{A t} v^{0}=\operatorname{sgn}\left(c_{0 j 2}\right) c_{0 j 2}>0, \forall t \geq 0 ;$ (a.3) $c_{0 j 1}=c_{0 j 2}=0$. Letting $\alpha_{1}, \alpha_{2}$ be arbitrary with $\alpha_{2} \neq 0$, we have $c^{T} \mathrm{e}^{A t} v^{0}=0, \forall t \geq 0$. Hence, $v^{0} \in \mathcal{A}_{\left(c^{T}, A\right)}$ in all the three cases. However, this is contradictory to $v^{0} \notin \mathcal{E}_{A}$ as $\alpha_{2} \neq 0$ in all the three cases.

Statement (b) follows from the fact that $E\left(\lambda_{i}\right) \cap \mathcal{X}_{c^{T}} \neq\{0\}$ for any nonzero real eigenvalue $\lambda_{i}$. Statement (c) follows from Lemma 4.7. The necessary and sufficient condition for (c) is a consequence of [13], Corollary 5.2 and Lemma 4.7. We finally consider (d). Without loss of generality, let $c_{0 j 1} \neq 0$. Letting $v^{0}=\operatorname{sgn}\left(c_{0 j 1}\right) \mathbf{e}_{0 j 1}$, it is easy to verify that $c^{T} v^{0}=\operatorname{sgn}\left(c_{0 j 1}\right) c_{0 j 1}>0$. Hence, $v^{0} \in \operatorname{int} \mathcal{X}_{c^{T}}$. By Proposition 4.10, we obtain (d).

"Sufficiency". If $c_{J_{0}}^{T} \neq 0$, the sufficiency follows readily from Proposition 4.10. Now consider the case where $c_{J_{0}}^{T}=0$. Suppose, in contrast, that a vector $v^{0}$ exists such that $v^{0} \notin \mathcal{E}_{A}$ but $v^{0} \in \mathcal{A}_{\left(c^{T}, A\right)}$. Let $v^{0}=\left(v^{J_{0}}, v_{c p}\right)$, where $v^{J_{0}}$ and $v_{c p}$ are the sub-vectors corresponding to the zero eigenvalue and the complex eigenvalues respectively. By (a) and $v^{0} \notin \mathcal{E}_{A}$, we have $v_{c p} \neq 0$. Notice that $c^{T} \mathrm{e}^{A t} v^{0}=c_{c p}^{T} \mathrm{e}^{J_{c p} t} v_{c p}$. By the observability condition in (c) and the argument for (ii) of Proposition 4.8, we conclude that a $t_{*}>0$ exists such that $c^{T} \mathrm{e}^{A t_{*}} v^{0} \equiv c_{c p}^{T} \mathrm{e}^{J_{c p} t_{*}} v_{c p}<0$. This results in a contraction to $v^{0} \in \mathcal{A}_{\left(c^{T}, A\right)}$.

Corollary 4.15. Let $\left(c^{T}, A\right)$ be an observable pair. Then $\mathcal{E}_{\left(c^{T}, A\right)}=\mathcal{A}_{\left(c^{T}, A\right)}$ if and only if the following conditions are all satisfied: (a) $A$ is of order greater than one; (b) if $A$ is of even order, then $A$ has complex eigenvalues only; (c) if $A$ is of odd order greater than one, then $A$ has no nonzero eigenvalue except the zero eigenvalue with algebraic multiplicity one and each complex eigenvalue of $A$ has a positive real part.

Proof. We show the necessity first. The statement (a) is trivial. The above mentioned observability results, e.g., [13], Corollary 5.2 and Lemma 4.7, along with (b) of Theorem 4.14, show that the block $J_{0}$ must have order at most one and that $A$ has no nonzero eigenvalue. Thus (i) if $A$ is of even order, then it has complex eigenvalues only; and (ii) if $A$ is of odd order greater than one, then it must have the zero eigenvalue of algebraic multiplicity one. Notice that $c_{J_{0}}^{T}$ is a nonzero scalar via the observability condition in case (ii). Hence in this case, 
each complex eigenvalue of $A$ has the positive real part by (d) of Theorem 4.14. The sufficiency follows readily from Theorem 4.14 and the observability condition.

Example 4.16. Consider a contact mechanical system involving a moving mass interacted with a spring modeled by the linear complementarity system (LCS): $\ddot{x}=u, 0 \leq u \perp x+u \geq 0$, where $x, u \in \mathbb{R}$. See Section 6.2 for more on LCSs. This model is equivalent to a planar bimodal CLS with

$$
A=\left[\begin{array}{ll}
0 & 1 \\
0 & 0
\end{array}\right], \quad b=\left(\begin{array}{l}
0 \\
1
\end{array}\right), \quad c=\left(\begin{array}{l}
1 \\
0
\end{array}\right), \quad A-b c^{T}=\left[\begin{array}{cc}
0 & 1 \\
-1 & 0
\end{array}\right] .
$$

It is easy to show that $\left(c^{T}, A\right)$ is an observable pair and that $A-b c^{T}$ has two complex eigenvalues but $A$ has no complex eigenvalue. By Corollary 4.15, a system trajectory starting from some non-equilibrium initial state has finitely many mode switchings in the forward time. Indeed, this system has at most one mode switching starting from any initial state.

An immediate consequence of Corollary 4.15 is that a bimodal CLS with infinite mode switchings and $\left(c^{T}, A\right)$ being an observable pair contains a continuum of equilibria if $n \geq 3$ is odd. Hence, such a CLS cannot be asymptotically stable at $x^{e}=0$ but may be semistable instead, where the notion of semistability is stronger than Lyapunov stability but weaker than asymptotic stability [5].

\section{CLSs without MOde SWitchings}

In this section, we derive necessary and sufficient conditions for the CLSs without mode switchings along any trajectory, which we simply call the switch-free CLSs. While a CLS of this type is perhaps the simplest, it is not equivalent to a smooth ODE system. Furthermore, characterizing such a CLS is non-trivial; for one thing, it involves the use of the simple switching property shown in Proposition 2.2.

Lemma 5.1. Consider the CLS (2.1). Suppose that for any $x^{0} \in \mathbb{R}^{n}$, there is no switching along $x\left(t, x^{0}\right)$ for all $t \geq 0$. Then the following statements hold:

(a) For a given $x^{0} \in \mathbb{R}^{n}, x\left(t, x^{0}\right) \in \mathcal{X}_{i}, \forall t \in(-\infty, \infty)$ for each $i \in \mathcal{J}\left(x^{0}\right)$. Moreover, $\mathcal{J}\left(x\left(t, x^{0}\right)\right)=$ $\mathcal{J}^{r}\left(x\left(t, x^{0}\right)\right)=\mathcal{J}\left(x^{0}\right)$ for all $t \geq 0$;

(b) If $x^{0} \in \operatorname{int} \mathcal{X}_{i}$, then $x\left(t, x^{0}\right) \in \mathcal{X}_{i}$ for all $t \in(-\infty, \infty)$;

(c) For each $i \in \mathcal{I}\left(x^{0}\right), x\left(t, x^{0}\right) \in \mathcal{X}_{i}$ for all $t \in(-\infty, \infty)$;

(d) $\mathcal{I}\left(x\left(t, x^{0}\right)\right)=\mathcal{I}\left(x^{0}\right)=\mathcal{J}\left(x^{0}\right)$ for all $t \in(-\infty, \infty)$;

(e) If $x^{0} \in \operatorname{int} \mathcal{X}_{i}\left(\right.$ resp. $\left.x^{0} \in \operatorname{bd} \mathcal{X}_{i}\right)$, then $x\left(t, x^{0}\right) \in \operatorname{int} \mathcal{X}_{i}$ (resp. $x\left(t, x^{0}\right) \in \operatorname{bd} \mathcal{X}_{i}$ ) for all $t \in(-\infty, \infty)$;

(f) If $x^{0} \in \operatorname{bd} \mathcal{X}_{i}$, then there exists $j \in\left\{1, \ldots, \ell_{i}\right\}$ such that $\left(C_{i} x\left(t, x^{0}\right)\right)_{j} \equiv 0$ for all $t \in(-\infty, \infty)$.

Proof. (a) We first show that $x\left(t, x^{0}\right) \in \mathcal{X}_{i}$ for all $t \geq 0$. Consider $i \in \mathcal{J}\left(x^{0}\right)$ and let $t_{*} \equiv \sup \left\{\bar{t} \geq 0 \mid x\left(t, x^{0}\right) \in \mathcal{X}_{i}\right.$, $\forall t \in[0, \bar{t}]\}$. Clearly, $t_{*}>0$. Suppose $t_{*}$ is finite. Then we have $i \notin \mathcal{J}\left(x\left(t_{*}, x^{0}\right)\right)$. However, by the uniqueness of the solution and the reverse-time property, we have $i \in \mathcal{J}^{r}\left(x\left(t_{*}, x^{0}\right)\right)$. This thus implies that $t_{*}$ is a switching time thanks to the simple switching property, which leads to a contradiction. We then show that $t=0$ is a non-switching time. Indeed, if it is false, then for any $\varepsilon>0, x\left(t, x^{r}\left(\varepsilon, x^{0}\right)\right)$ has a switching at $t=\varepsilon$, which is a contradiction. Hence, we have $\mathcal{J}^{r}\left(x^{0}\right)=\mathcal{J}\left(x^{0}\right)$. This equality, together with Proposition 2.2 and a similar argument for $t \geq 0$, implies that $\mathcal{J}^{r}\left(x\left(t, x^{0}\right)\right)=\mathcal{J}\left(x^{0}\right)$ for all $t \geq 0$. This completes the proof for (a).

(b) If $x^{0} \in \operatorname{int} \mathcal{X}_{i}$, then we have $\mathcal{J}\left(x^{0}\right)=\mathcal{J}^{r}\left(x^{0}\right)=\{i\}$. We then obtain the desired result from (a).

(c) Consider $i \in \mathcal{I}\left(x^{0}\right)$. Since $\mathcal{X}_{i}$ is of full dimension, there is a vector $y \in \operatorname{int} \mathcal{X}_{i}$, i.e., $C_{i} y>0$, with $y \neq x^{0}$. By the convexity of the cone $\mathcal{X}_{i}$, we have $x^{0}+\varepsilon\left(y-x^{0}\right) \in \operatorname{int} \mathcal{X}_{i}$ for all $\varepsilon \in(0,1]$. Hence, we deduce from statement (b) that $x\left(t, x^{0}+\varepsilon\left(y-x^{0}\right)\right) \in \mathcal{X}_{i}$, or equivalently $C_{i} x\left(t, x^{0}+\varepsilon\left(y-x^{0}\right)\right) \equiv C_{i} \mathrm{e}^{A_{i} t}\left[x^{0}+\varepsilon\left(y-x^{0}\right)\right] \geq 0$, 
for all $t \in(-\infty, \infty)$ and $\varepsilon \in(0,1]$. Letting $\varepsilon \downarrow 0$, we see that $C_{i} x\left(t, x^{0}\right) \equiv C_{i} \mathrm{e}^{A_{i} t} x^{0} \geq 0$ for all $t \in(-\infty, \infty)$. This completes the proof for $(\mathrm{c})$.

(d) Clearly, $\mathcal{I}\left(x\left(t, x^{0}\right)\right) \supseteq \mathcal{I}\left(x^{0}\right)$ as shown in (c). We only need to show $\mathcal{I}\left(x\left(t, x^{0}\right)\right) \subseteq \mathcal{I}\left(x^{0}\right)$. Notice that (c) implies $\mathcal{I}\left(x^{0}\right) \subseteq \mathcal{J}\left(x\left(t, x^{0}\right)\right) \equiv \mathcal{J}\left(x^{0}\right)$. Hence, due to $\mathcal{J}\left(x^{0}\right) \subseteq \mathcal{I}\left(x^{0}\right)$, we have $\mathcal{J}\left(x^{0}\right)=\mathcal{I}\left(x^{0}\right)$. Replacing $x^{0}$ by $x\left(t, x^{0}\right)$, via the initial state shifting argument, and noting (a), we obtain (d).

(e) We prove the case where $x^{0} \in \operatorname{int} \mathcal{X}_{i}$; the other case follows from the similar argument. Note that if $x^{0} \in \operatorname{int} \mathcal{X}_{i}$, then $\mathcal{J}\left(x^{0}\right)=\mathcal{J}^{r}\left(x^{0}\right)=\{i\}$. This yields from $(\mathrm{d})$ that $\mathcal{I}\left(x\left(t, x^{0}\right)\right)=\{i\}$ for all $t \in \mathbb{R}$. This shows that $x\left(t, x^{0}\right) \notin \mathrm{bd} \mathcal{X}_{i}$ for all $t$. Thus $x\left(t, x^{0}\right)$ must be in the interior of $\mathcal{X}_{i}$ for all $t$.

(f) Since $x^{0} \in \operatorname{bd} \mathcal{X}_{i} \subseteq \mathcal{X}_{i}$, we deduce from (d) that $i \in \mathcal{J}\left(x^{0}\right)$. Hence, there exists a scalar $\varepsilon>0$ such that $C_{i} \mathrm{e}^{A_{i} t} x^{0} \geq 0$ for all $t \in[0, \varepsilon]$. By Cayley-Hamilton Theorem, we further see that for each $j \in\left\{1, \ldots, \ell_{i}\right\}$, either $\left(C_{i}\right)_{j} \bullet \mathrm{e}^{A_{i} t} x^{0} \equiv 0$ or $\left(C_{i}\right)_{j} \bullet \mathrm{e}^{A_{i} t} x^{0}>0$ for all $t \in(0, \varepsilon]$ with $\varepsilon>0$ appropriately restricted. Note that there is at least one $j$ satisfying $\left(C_{i}\right)_{j} \bullet \mathrm{e}^{A_{i} t} x^{0} \equiv 0, \forall t \in(0, \varepsilon]$, since otherwise, $C_{i} x\left(t, x^{0}\right)>0$ on $(0, \varepsilon]$, i.e. $x\left(t, x^{0}\right)=\mathrm{e}^{A_{i} t} x^{0}$ is in the interior of $\mathcal{X}_{i}$ on $(0, \varepsilon]$. This leads to $x^{0} \in \operatorname{int} \mathcal{X}_{i}$ according to (e), which is contradictory to the assumption that $x^{0} \in \operatorname{bd} \mathcal{X}_{i}$. Furthermore, $\left(C_{i}\right)_{j} \bullet \mathrm{e}^{A_{i} t} x^{0} \equiv 0, \forall t \in(0, \varepsilon]$ implies that $\left(C_{i} x^{0}\right)_{j}=0$ and $x^{0} \in \bar{O}\left(\left(C_{i}\right)_{j} \bullet, A_{i}\right)$. Hence, $\left(C_{i} \mathrm{e}^{A_{i} t} x^{0}\right)_{j} \equiv 0, \forall t \in(-\infty, \infty)$.

Proposition 5.2. The following statements are equivalent for the CLS (2.1):

(a) the CLS has no switching along $x\left(t, x^{0}\right)$ for all $t \geq 0$ and any $x^{0} \in \mathbb{R}^{n}$;

(b) for each $i=1, \ldots, m$, there exists $G^{i}: \mathbb{R} \rightarrow \mathbb{R}_{+}^{\ell_{i} \times \ell_{i}}$ such that $C_{i} \mathrm{e}^{A_{i} t}=G^{i}(t) C_{i}$ for all $t \geq 0$ and each $G^{i}(t)$ has no vanishing rows;

(c) $\mathcal{A}_{i}=\mathcal{X}_{i}$ for all $i=1, \ldots, m$;

(d) $\bigcup_{i=1}^{m} \mathcal{A}_{i}=\mathbb{R}^{n}$

(e) for any $x \in \mathbb{R}^{n}$ and any $i \in \mathcal{I}(x), A_{i} x \in \mathcal{T}\left(x ; \bigcap_{i \in \mathcal{I}(x)} \mathcal{X}_{i}\right)$.

Moreover, if any one of (a)-(e) holds, then $\mathcal{A}_{i}=\widehat{\mathcal{A}_{i}}$ and $\bar{O}\left(C_{i}, A_{i}\right)=\operatorname{ker}\left(C_{i}\right)$ for each $i=1, \ldots, m$.

Proof. (b) $\Rightarrow$ (a). This is trivial, since if such a $G^{i}$ exists for each piece $\mathcal{X}_{i}$, then for any $x^{0}$ such that $x^{0} \in \mathcal{X}^{i}$, we have $C_{i} \mathrm{e}^{A_{i} t} x^{0}=G^{i}(t) C_{i} x^{0} \geq 0, \forall t \geq 0$. Hence, $x\left(t, x^{0}\right) \equiv \mathrm{e}^{A_{i} t} x^{0}$ is the unique solution starting from $x^{0}$ and $x\left(t, x^{0}\right) \in \mathcal{X}_{i}$ for all $t \geq 0$. Thus there is no mode switching along $x\left(t, x^{0}\right)$.

$(\mathrm{a}) \Rightarrow(\mathrm{b})$. Suppose (a) holds. Then for any $x^{0} \in \mathcal{X}_{i}$, we deduce from (d) of Lemma 5.1 that $x\left(t, x^{0}\right) \in \mathcal{X}_{i}$ for all $t \geq 0$. Letting $\left(C_{i}\right)_{j}$ denote the $j$ th row of $C_{i}$, we thus have the following implication:

$$
\forall x^{0} \text { such that } C_{i} x^{0} \geq 0 \Rightarrow\left(C_{i}\right)_{j} \bullet \mathrm{e}^{A_{i} t} x^{0} \geq 0, \quad \forall t \geq 0
$$

For any fixed $t \geq 0$, we thus conclude, via Farkas' Lemma, that there exists $z_{j}(t) \in \mathbb{R}_{+}^{\ell_{i}}$ such that $\left(C_{i}\right)_{j} \mathrm{e}^{A_{i} t}=$ $z_{j}^{T}(t) C_{i}$. Letting $G_{j \bullet}^{i}(t)=z_{j}^{T}(t)$, we obtain the required $G^{i}(t)$. To show that $G_{j \bullet}^{i}(t) \supsetneqq 0, j=1, \ldots, \ell_{i}$ for all $t \geq 0$, we choose $x^{0} \in \operatorname{int} \mathcal{X}_{i}$. Therefore, by (e) of Lemma 5.1, we have $C_{i} x\left(t, x^{0}\right) \equiv C_{i} \mathrm{e}^{A_{i} t} x^{0}>0$ for all $t \geq 0$. Combining this with $C x^{0}>0$, we obtain the desired result.

(a) $\Leftrightarrow(\mathrm{c}) \Leftrightarrow(\mathrm{d})$. We show $(\mathrm{a}) \Leftrightarrow(\mathrm{c})$ first. Clearly, $\mathcal{A}_{i} \subseteq \mathcal{X}_{i}$. The opposite inclusion holds due to (c) of Lemma 5.1, which also shows (a) $\Rightarrow(\mathrm{d})$. Furthermore, (d) implies that any $x^{0} \in \mathbb{R}^{n}$ must be in $\mathcal{A}_{i}$ for some $i$, which shows that $x\left(t, x^{0}\right)$ is switching free for all $t \geq 0$. Hence, $(\mathrm{d}) \Rightarrow$ (a) holds.

(a) $\Leftrightarrow(\mathrm{e})$. The $\Rightarrow$ direction is simply an implication of Lemma 5.1(d). To show the other direction, we notice that for any $x \in \mathcal{X}_{i} \cap \mathcal{X}_{j}$, we have $x \in \mathcal{X}_{i} \cap \mathcal{X}_{j} \Rightarrow A_{i} x \in \mathcal{X}_{i} \cap \mathcal{X}_{j} \Rightarrow A_{i}^{2} x \in \mathcal{X}_{i} \cap \mathcal{X}_{j} \Rightarrow \ldots$ 
Finally, $\mathcal{A}_{i}=\widehat{\mathcal{A}_{i}}$ holds due to (3.2), (c) of Lemma 5.1 and (c) of Proposition 5.2. To see $\operatorname{ker}\left(C_{i}\right)=\bar{O}\left(C_{i}, A_{i}\right)$ for each $i=1, \ldots, m$, we notice that $\bar{O}\left(C_{i}, A_{i}\right) \subseteq \operatorname{ker}\left(C_{i}\right)$. On the other hand, if $x^{0} \in \operatorname{ker}\left(C_{i}\right)$, we have $C_{i} \mathrm{e}^{A_{i} t} x^{0}=G^{i}(t) C_{i} x^{0} \equiv 0$ for all $t$. Thus $x^{0} \in \bar{O}\left(C_{i}, A_{i}\right)$. This completes the proof.

Remark 5.3. In Corollary 2.11, Chapter 4 of [4], the following result is proved. For a closed convex solid cone $\mathcal{X} \subseteq \mathbb{R}^{n}$ (not necessarily polyhedral) and a matrix $A \in \mathbb{R}^{n \times n},\left[x^{0} \in \mathcal{X} \Rightarrow \mathrm{e}^{A t} x^{0} \in \mathcal{X}\right.$ for all $\left.t \geq 0\right]$ if and only if $y^{T} A x \geq 0$ for all pairs $(x, y)$ satisfying $\mathcal{X} \ni x \perp y \in \mathcal{X}^{*}$, where $\mathcal{X}^{*}$ is the dual cone of $\mathcal{X}$. (This result holds for a polyhedral cone $\mathcal{X}$ even without the solidness assumption.) Specializing this result to each cone $\mathcal{X}_{i}$, we obtain the equivalence between (c) and (e) in Proposition 5.2. This characterization result further shows finite verification of a switch-free CLS by making use of the polyhedral structure of the CLS. Indeed, since each dual cone $\mathcal{X}_{i}^{*}$ remains polyhedral, we have $\mathcal{X}_{i}^{*}=\left\{y \mid \widetilde{C}_{i} y \geq 0\right\}$ for some matrix $\widetilde{C}_{i}$. Hence, the implication $\left[\mathcal{X}_{i} \ni x \perp y \in \mathcal{X}_{i}^{*}\right] \Rightarrow y^{T} A_{i} x \geq 0$ holds if and only if the semi-algebraic set $\left\{(x, y) \in \mathbb{R}^{n} \times \mathbb{R}^{n} \mid C_{i} x \geq 0\right.$, $\left.\widetilde{C}_{i} y \geq 0, x^{T} y=0, y^{T} A_{i} x<0\right\}$ is empty. The latter problem can be verified via the Tarski-Seidenberg decision procedure in finite steps as discussed in Section 4.1.1.

Corollary 5.4. A bimodal $C L S: \dot{x}=A x+b \max \left(0, c^{T} x\right)$, where $A \in \mathbb{R}^{n \times n}$ and $b$ and $c$ are two $n$-vectors with $c \neq 0$, is switching free along any trajectory if and only if $c$ is an eigenvector of the matrix $A^{T}$.

Proof. To show the "if" part, let $c$ be an eigenvector of $A^{T}$, i.e., $A^{T} c=\lambda c$ for some $\lambda \in \mathbb{R}$. Therefore, $\left(A+b c^{T}\right)^{T} c=\left(\lambda+b^{T} c\right) c$, namely, $c$ is an eigenvector of $\left(A+b c^{T}\right)^{T}$. Hence, $c^{T} \mathrm{e}^{A t}=\mathrm{e}^{\lambda t} c^{T}$ and $c^{T} \mathrm{e}^{\left(A+b c^{T}\right) t}=$ $\mathrm{e}^{\left(\lambda+b^{T} c\right) t} c^{T}$ for all $t$. Letting $G_{1}(t)=\mathrm{e}^{\left(\lambda+b^{T} c\right) t}, G_{2}(t)=\mathrm{e}^{\left(\lambda+b^{T} c\right) t}$ and using Proposition 5.2, we conclude that the bimodal CLS has no switching. Conversely, for a switch-free bimodal CLS, we deduce, via Proposition 5.2, that there exists a real-valued function $g(t)$ such that $c^{T} \mathrm{e}^{A t}=g(t) c^{T}$ for all $t \geq 0$. Recalling $c \neq 0$, we obtain $g(t)=\frac{c^{T} \mathrm{e}^{A t} c}{c^{T} c}$. This shows that $g$ is smooth in $t$. Consequently, $c^{T} A \mathrm{e}^{A t}=\dot{g}(t) c^{T}$ for all $t \geq 0$. Letting $t=0$, we have $c^{T} A=\dot{g}(0) c^{T}$ as desired.

Remark 5.5. Note that the characterizing condition in Corollary 5.4 does not involve $b$.

\section{Asymptotic stability of CLSs And LCSs}

Stability analysis of hybrid and switched dynamical systems has received tremendous interest in the systems and control community over the past few years $[16,19,20,23]$. In the setting of complementarity and related systems, the recent paper [10] extensively studies Lyapunov stability of the LCSs with Lipschitz right-hand sides, or the simply called Lipschitz LCSs. However, a critical question left open in [10] is whether asymptotic stability is equivalent to its exponential stability. The major difficulty in investigating the connection between the two stability concepts for LCSs is due to state dependent mode switchings to be discussed soon. In spite of this difficulty, the present section provides an affirmative answer to this question, and thus bridges the gap between the two stability notions. The significance of this result lies in two aspects: (i) This result validates the Lyapunov stability analysis in [10] via the converse Lyapunov Theorem; see [22] for more discussions on the related converse Lyapunov Theorems. Besides, it broadens stability analysis of nonlinear complementarity systems via the first-order approximation approach. (ii) Exponential stability is more desirable than asymptotic stability in that it is more robust when a system is subject to disturbances and/or unknown dynamics. Hence, this equivalence result facilitates the verification of exponential stability since one only needs to check less restrictive conditions for asymptotic stability. 


\subsection{Preliminary discussion}

It is well known that asymptotic stability does not imply exponential stability for general nonlinear systems, even for piecewise linear systems with or without discontinuous right-hand sides [19], Example 1. It has been observed in [19] that a switched linear system, with either a continuous or a discontinuous right-hand side, is related to a linear time-varying system $\dot{x}=A(t) x$, although the two classes of systems are quite different in general. For linear time-varying systems, uniform asymptotic stability [21], Definition 3.2, plays an important role that relates asymptotic stability to exponential stability [13], Theorem 8.10. Roughly speaking, uniform asymptotic stability requires a state trajectory converge to an equilibrium independent of initial times. As a matter of fact, if $A(t)$ is (piecewise) continuous, then uniform asymptotic stability of $x^{e}=0$ is equivalent to its exponential stability [21], Theorem 3.9. It is further shown in [19], Lemma 1, that if a switched linear system is subject to state independent switchings, then uniform asymptotic stability and exponential stability are equivalent. However, asymptotic stability of such a switched linear system does not always imply uniform asymptotic stability as illustrated in [19], Example 2. The relations between these stability concepts can be summarized in the following diagram:

- linear time-invariant systems:

asymptotic stability $\Leftrightarrow$ uniform asymptotic stability $\Leftrightarrow$ exponential stability;

- linear time-varying systems:

asymptotic stability $\nRightarrow$ uniform asymptotic stability $\Leftrightarrow$ exponential stability;

- switched linear systems with state independent switchings:

asymptotic stability $\nRightarrow$ uniform asymptotic stability $\Leftrightarrow$ exponential stability;

- switched linear systems with state dependent switchings:

asymptotic stability $\nRightarrow$ uniform asymptotic stability $\nRightarrow$ exponential stability.

It is shown in [19] that switching rules are crucial to (resp. asymptotic or uniform asymptotic) stability of switched linear systems, even when the switchings are state independent. Certain "regularity conditions" on time intervals between switchings have to be imposed to guarantee asymptotic stability for such a system, even if each mode is asymptotically stable.

\subsection{Uniform asymptotic and exponential stability}

In this subsection, we show that asymptotic stability of the CLS (2.1) does imply exponential stability, despite the fact that switchings are state dependent. A key step is to show uniform asymptotic stability based upon Lipschitz continuity of the CLS. This observation is motivated by [36], Lemma 8.2 (originally due to [26]), which considers a class of (closed and convex valued) linear differential inclusions. By using the Lipschitz continuity of the CLS, we obtain the same uniform stability property via a simpler argument. We begin with a slightly general setting.

Proposition 6.1. Consider the time-invariant system on $\mathbb{R}^{n}: \dot{x}=f(x)$ with $x^{e}=0$ as its equilibrium. Assume that $f: \mathbb{R}^{n} \rightarrow \mathbb{R}^{n}$ is Lipschitz continuous at $x^{e}=0$ and the system is asymptotically stable at $x^{e}=0$. Then for any sufficiently small $\delta>0$ and a given scalar $0<c<1$, there is a scalar $T_{\delta, c}>0$ (depending on $\delta$ and $c$ only) such that the following implication holds:

$$
\left\|x^{0}\right\| \leq \delta \Rightarrow\left\|x\left(t, x^{0}\right)\right\| \leq c \delta, \forall t \geq T_{\delta, c}
$$

Proof. Since the system is asymptotically stable at $x^{e}=0$, it is stable at $x^{e}=0$ and there is a $r_{1}>0$ such that $\left\|x^{0}\right\| \leq r_{1} \Rightarrow \lim _{t \rightarrow \infty} x\left(t, x^{0}\right)=0$. Moreover, by properly restricting $r_{1}$, we have a scalar $r_{2}>0$ such that $\left\|x^{0}\right\| \leq r_{1} \Rightarrow\left\|x\left(t, x^{0}\right)\right\| \leq r_{2}, \forall t \geq 0$, due to the stability at $x^{e}$, and that $f(\cdot)$ is Lipschitz continuous 
on the closed ball $\mathcal{B}_{r_{2}}$, i.e. a constant $L>0$ exists such that $\left\|f\left(x^{1}\right)-f\left(x^{2}\right)\right\| \leq L\left\|x^{1}-x^{2}\right\|$ for any $x^{1}, x^{2} \in \mathcal{B}_{r_{2}}$. The latter further implies that for any $x^{0}, \widehat{x}^{0} \in \mathcal{B}_{r_{1}}$,

$$
\left\|x\left(t, x^{0}\right)-x\left(t, \widehat{x}^{0}\right)\right\| \leq \mathrm{e}^{L t}\left\|x^{0}-\widehat{x}^{0}\right\|, \forall t \geq 0 .
$$

Now consider any given $0<\delta<r_{1}$ and $0<c<1$. Suppose the proposition does not hold for such the $\delta$ and $c$, then we have an initial state sequence $\left\{x_{k}^{0}\right\} \subseteq \mathcal{B}_{\delta}$ and a nondecreasing time sequence $\left\{t_{k}\right\}$ with $\lim _{k \rightarrow \infty} t_{k}=\infty$ such that $\left\|x\left(t_{k}, x_{k}^{0}\right)\right\|>c \delta$. We further deduce from the stability of $x^{e}=0$ that a scalar $\mu>0$ exists (with $\mu<\delta$ ) such that $x^{0} \in \mathcal{B}_{\mu} \Rightarrow\left\|x\left(t, x^{0}\right)\right\| \leq c \delta, \forall t \geq 0$. By the similar time shifting argument as in [36], Lemma 8.2, we have $\left\|x\left(t, x_{k}^{0}\right)\right\| \geq \mu$ for all $t \in\left[0, t_{k}\right]$. Moreover, let $x^{*} \in \mathcal{B}_{\delta}$ be an accumulation point of the sequence $\left\{x_{k}^{0}\right\}$ (the existence of $x^{*}$ is due to compactness of $\mathcal{B}_{\delta}$ ). Without loss of generality, let $\left\{x_{k}^{0}\right\}$ be the subsequence of itself that converges to $x^{*}$. We claim that $\left\|x\left(t, x^{*}\right)\right\| \geq \mu, \forall t \geq 0$. Suppose the contrary, i.e. a time $t_{*} \geq 0$ exists such that $\left\|x\left(t_{*}, x^{*}\right)\right\|<\mu$. Let $\varepsilon:=\mu-\left\|x\left(t_{*}, x^{*}\right)\right\|>0$. Hence, there is a sufficiently large $N$ such that $\left\|x_{N}^{0}-x^{*}\right\| \leq \frac{\varepsilon}{2 \mathrm{e}^{L t_{*}}}$ and $t_{N} \geq t_{*}$; the latter implies $\left\|x\left(t_{*}, x_{N}^{0}\right)\right\| \geq \mu$. Using (6.2), we have $\left\|x\left(t_{*}, x_{N}^{0}\right)-x\left(t_{*}, x^{*}\right)\right\| \leq \varepsilon / 2$. This in turn shows $\left\|x\left(t_{*}, x_{N}^{0}\right)\right\| \leq \mu-\varepsilon / 2<\mu$, but this is a contradiction. Therefore, the claim that $\left\|x\left(t, x^{*}\right)\right\| \geq \mu, \forall t \geq 0$ holds. However, this leads to another contradiction because $\lim _{t \rightarrow \infty} x\left(t, x^{*}\right)=0$ as $x^{*} \in \mathcal{B}_{\delta} \subseteq \mathcal{B}_{r_{1}}$. As a result, we conclude that the proposition holds.

Remark 6.2. The implication (6.1) leads to uniform asymptotic stability of a time-invariant system readily. This result may be extended to a time-varying system with $f(t, x)$ satisfying local Lipschitz condition in $x$, i.e., for any $t \geq 0,\left\|f\left(t, x^{1}\right)-f\left(t, x^{2}\right)\right\| \leq L\left\|x^{1}-x^{2}\right\|$ for any $x^{1}, x^{2}$ in a closed ball of the equilibrium. However, in order to obtain uniform asymptotic stability, one needs to further show that the scalar $T_{\delta, c}$ is independent of initial times. See [21], Section 4.3 for the detailed discussions. Finally, we notice that the system [19], Example 1, satisfies the uniform asymptotic property, though its right-hand side is not continuous near $x^{e}=0$. This suggests that the Lipschitz condition is only sufficient and may be relaxed; see the generalization discussed at this end of this section.

We now return to the CLS (2.1), which clearly possesses a time-invariant right-hand side. The following facts have been given in [10,11]: (i) the right-hand side of the CLS is globally Lipschitz continuous; (ii) a state trajectory of the CLS is positively homogeneous, i.e., $x\left(t, \tau x^{0}\right)=\tau x\left(t, x^{0}\right), \forall t \geq 0$, for any $\tau \geq 0$ and $x^{0} \in \mathbb{R}^{n}$; (iii) local (resp. asymptotic/exponential) stability of the CLS implies its global (resp. asymptotic/exponential) stability. Putting these facts and Proposition 6.1 together, we immediately conclude that if the CLS (2.1) is asymptotically stable at $x^{e}=0$, then for any scalars $\delta>0$ and $0<c<1$, there is a scalar $T_{\delta, c}>0$ such that the implication (6.1) holds. This property, together with the time-invariance and linear structure of the CLS, e.g., positive homogeneity, yields equivalence of asymptotic and exponential stability; see Theorem 6.3 below. The proof for this result is similar to that of [36], Lemma 8.2, which is generalized for linear time-varying systems in [21], Theorem 3.9, and [13], Theorem 8.10. To be self-contained, we include the proof in the Appendix.

Theorem 6.3. The CLS (2.1) is asymptotically stable at $x^{e}=0$ if and only if it is exponentially stable at $x^{e}=0$.

One of the important consequences of Theorem 6.3 is that it ensures existence of a Lyapunov function for an asymptotically stable CLS, via the converse Lyapunov theorem given in [10], Theorem 5.2. Recall that the right-hand side of the CLS (2.1) is Lipschitz continuous and directionally differentiable in $\mathbb{R}^{n}$. Consequently, by applying [10], Theorem 5.2, and the linear structure of the CLS, we have:

Corollary 6.4. Let $f(x)$ be the right-hand side of the CLS (2.1). The CLS is asymptotically stable at $x^{e}=0$ if and only if there exist positive constants $c_{1}, c_{2}, c_{3}, c_{4}$ and a Lipschitz continuous and directionally differentiable 
function $V$ such that the following conditions hold: (i) $c_{1}\|x\|^{2} \leq V(x) \leq c_{2}\|x\|^{2}, \forall x \in \mathbb{R}^{n}$; (ii) $V^{\prime}(x ; f(x)) \leq$ $-c_{3}\|x\|^{2}, \forall x \in \mathbb{R}^{n}$, where $V^{\prime}(x ; d)$ denotes the (one-sided) directional derivative of $V$ along the direction $d \in \mathbb{R}^{n}$ at $x$; and (iii) $\left|V^{\prime}(x ; z)-V^{\prime}(x ; \widetilde{z})\right| \leq c_{4}\|x\|\|z-\widetilde{z}\|$ for all $x, z, \widetilde{z} \in \mathbb{R}^{n}$.

The equivalence result established above allows us to obtain better tests for asymptotic/exponential stability of a nonlinear nonsmooth system via linearization (or Lyapunov's indirect method). Specifically, consider an ODE system $\mathbf{I}: \dot{x}=f(x)$, where $x^{e} \in \mathbb{R}^{n}$ is an equilibrium and $f: \mathbb{R}^{n} \rightarrow \mathbb{R}^{n}$ is a (continuous and) piecewise $\mathrm{C}^{1}$-function on a neighborhood of $x^{e}$ [31]. It is known that such an $f$ is B-differentiable at $x^{e}$ [10]. Let the "first-order approximated system" of system I be $\mathbf{I I}: \dot{z}=f^{\prime}\left(x^{e} ; z\right)$, where $f^{\prime}\left(x^{e} ; z\right)$ is the directional derivative of $f$ at $x^{e}$ along $z$. Since $f^{\prime}\left(x^{e} ; \cdot\right)$ is (continuous and) piecewise linear and positively homogeneous, we see from Theorem 6.3 that asymptotic stability of system II at $z^{e}=0$ is equivalent to its exponential stability. Hence, by [10], Corollary 5.6, asymptotic stability of system II at $z^{e}=0$ implies asymptotic and exponential stability of the original system $\mathbf{I}$ at $x^{e}$ as well as existence of a B-differentiable Lyapunov function for stability analysis. The following diagram summarizes this discussion:

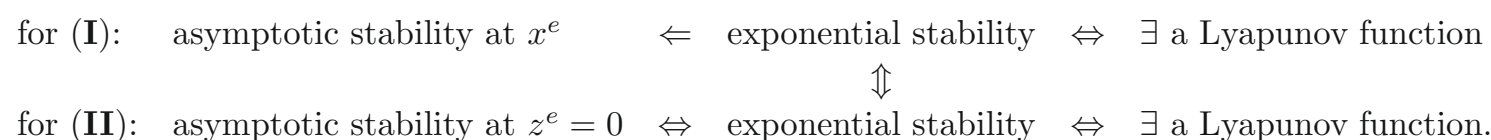

It should be mentioned that asymptotic stability of system $\mathbf{I}$ implies neither that of the approximated system II nor its stability, even for smooth systems.

Before ending this section, we briefly discuss extension to non-Lipschitz linear complementarity systems (LCSs). Given a vector $q \in \mathbb{R}^{m}$ and a matrix $M \in \mathbb{R}^{m \times m}$, its associated linear complementarity problem, denoted by $\operatorname{LCP}(q, M)$, is to find $u \in \mathbb{R}^{m}$ such that

$$
0 \leq u \perp q+M u \geq 0
$$

where, for two vectors $a$ and $b, a \perp b$ means that $a$ and $b$ are orthogonal, i.e., $a^{T} b=0$. See the monograph [14] for more details. Being a dynamic extension of the static $\operatorname{LCP}$, the $\operatorname{LCS}(A, B, C, D)$ is given by:

$$
\dot{x}=A x+B u, \quad 0 \leq u \perp C x+D u \geq 0
$$

where $x \in \mathbb{R}^{n}, u \in \mathbb{R}^{m},(A, B, C, D)$ is a matrix tuple of compatible dimensions, and we use $\operatorname{SOL}(C x, D)$ to represent the solution set of $\operatorname{LCP}(C x, D)$ for a given $x$ in the subsequent development. Apparently, an LCS is time-invariant and positively homogeneous. Recall that a key step in establishing the stability equivalence of the CLS is the implication of uniform asymptotic stability from asymptotic stability via the solution dependence and time invariance properties. This implication and positive homogeneity lead to exponential stability; see the following diagram for illustration:

$$
\begin{gathered}
\begin{array}{c}
\text { asymptotic } \\
\text { stability }
\end{array} \\
\text { sol. depend. }+ \text { time invar. }
\end{gathered} \begin{gathered}
\text { uniform } \\
\text { asymptotic stability }
\end{gathered} \stackrel{\text { pos. homogeneity }}{\Longrightarrow} \begin{gathered}
\text { exponential } \\
\text { stability. }
\end{gathered}
$$

We will extend this line of argument to a class of non-Lipschitz LCSs. In particular, we consider linear passive complementarity systems (LPCSs) [9]. $\operatorname{An} \operatorname{LCS}(A, B, C, D)$ is an LPCS if the corresponding linear system $(A, B, C, D)$ satisfies the passivity condition in term of the supply rate $u^{T} y$, where $u$ is treated as an input and $y \equiv C x+D u$ as an output, i.e., there exists a storage function $V: \mathbb{R}^{n} \rightarrow \mathbb{R}_{+}$such that $V\left(x^{0}\right)+$ $\int_{0}^{t} u^{T}\left(\tau, x^{0}\right)\left[C x\left(\tau, x^{0}\right)+D u\left(\tau, x^{0}\right)\right] \mathrm{d} \tau \geq V\left(x\left(t, x^{0}\right)\right), \quad \forall t \geq 0$ for any $x^{0} \in \mathbb{R}^{n}$ and any $L^{2}$-solution pair $\left(x\left(t, x^{0}\right), u\left(t, x^{0}\right)\right)$ of the LCS. It is known from linear system theory that if $(A, B, C, D)$ is a minimal realization, 
then the passivity condition is equivalent to the existence of a quadratic storage function $V(x)=\frac{1}{2} x^{T} K x$, where $K$ is a symmetric positive definite matrix satisfying the matrix inequality:

$$
\left[\begin{array}{cc}
A^{T} K+K A & K B-C^{T} \\
B^{T} K-C & -\left(D+D^{T}\right)
\end{array}\right] \quad \text { is negative semidefinite. }
$$

It is further shown in [9] that under the minimal realization assumption, the LPCS possesses a unique $L^{2}$-state solution $x\left(\cdot, x^{0}\right)$ on $[0, \infty)$, provided that $x^{0}$ is feasible, i.e., $\mathrm{SOL}\left(C x^{0}, D\right) \neq \emptyset$. Moreover, under this assumption, one can show, via the similar argument as [9], that a scalar $\mu>0$ exists such that for any feasible initial states $x^{0}, \widetilde{x}^{0}$ in $\mathbb{R}^{n},\left\|x\left(t, x^{0}\right)-x\left(t, \widetilde{x}^{0}\right)\right\| \leq \mu\left\|x^{0}-\widetilde{x}^{0}\right\|, \forall t \geq 0$. With this global solution dependence result and the fact that $\left\|x\left(t, x^{0}\right)\right\| \leq \mu\left\|x^{0}\right\|, \forall t \geq 0$ for any feasible $x^{0}$, we obtain:

Corollary 6.5. Assume that the $\operatorname{LPCS}(A, B, C, D)$ is asymptotically stable at $x^{e}=0$, where $(A, B, C, D)$ is a minimal realization. Then for any scalars $\delta>0$ and $0<c<1$, there is a scalar $T_{\delta, c}>0$ such that $\left[\left\|x^{0}\right\| \leq \delta\right.$ and $\left.S O L\left(C x^{0}, D\right) \neq \emptyset\right] \Rightarrow\left\|x\left(t, x^{0}\right)\right\| \leq c \delta, \forall t \geq T_{\delta, c}$. Moreover, the LPCS is exponentially stable at $x^{e}=0$.

\subsection{Stability of switch-free CLSs}

In this subsection, necessary and sufficient conditions are derived for the stability of a switch-free CLS by making use of its no switching property. In particular, stability of this class of CLSs is equivalent to that of its pieces and to the existence of a (continuous) piecewise quadratic Lyapunov function.

Theorem 6.6. Consider the switch-free CLS (2.1). The following statements are equivalent:

(a) the system is asymptotically/exponentially stable at $x^{e}=0$;

(b) each $A_{i}$ is Hurwitz for $i=1, \ldots, m$, i.e., each eigenvalue of $A_{i}$ has the negative real part;

(c) there exist symmetric positive definite matrices $\left\{Q_{i}\right\}_{i=1}^{m}$ such that $x^{T} Q_{i} x=x^{T} Q_{j} x$ for any $x \in \mathcal{X}_{i} \cap \mathcal{X}_{j}$, and $-\left(Q_{i} A_{i}+A_{i}^{T} Q_{i}\right)$ is strictly copositive on $\mathcal{X}_{i}$.

Proof. (a) $\Rightarrow$ (b). We focus on a pair $\left(C_{i}, A_{i}\right)$ and drop the subscript for notational simplicity. Since the cone $\mathcal{X}_{C}$ is solid, there exists a vector $x^{*} \in \operatorname{int} \mathcal{X}_{C}$. Hence, we deduce the existence of a real number $\varepsilon>0$ such that $v^{i} \equiv x^{*}+\varepsilon \mathbf{e}_{i} \in \operatorname{int} \mathcal{X}_{C}$ for all $i=1, \ldots, n$, where $\left\{\mathbf{e}_{i}\right\}_{i=1}^{n}$ is the standard basis for $\mathbb{R}^{n}$. It is easy to verify that the set $\left\{v^{i}\right\}_{i=1}^{n}$ is linear independent and thus forms a basis for $\mathbb{R}^{n}$. By Lemma 5.1, $x\left(t, v^{i}\right)=\mathrm{e}^{A t} v^{i}, \forall t \geq 0$. Moreover, asymptotic stability of the CLS implies $\lim _{t \rightarrow \infty} x\left(t, v^{i}\right) \equiv \lim _{t \rightarrow \infty} \mathrm{e}^{A t} v^{i}=0$ for each $i=1, \ldots, n$. We further notice that any $x \in \mathbb{R}^{n}$ is a linear combination of $\left\{v^{i}\right\}_{i=1}^{n}$. Consequently, $\lim _{t \rightarrow \infty} \mathrm{e}^{A t} x=0$ for all $x \in \mathbb{R}^{n}$. This shows that the matrix $A$ is Hurwitz.

(b) $\Rightarrow$ (a). For any $x^{0} \in \mathbb{R}^{n}, x\left(t, x^{0}\right)=\mathrm{e}^{A_{i} t} x^{0}, \forall t \geq 0$ remains in a piece whose dynamics is defined by some $A_{i}$. Since each $A_{i}$ is Hurwtiz, $\left\|\mathrm{e}^{A_{i} t}\right\|$ is bounded for all $t \geq 0$. Therefore, $\left\|x\left(t, x^{0}\right)\right\| \leq\left\|\mathrm{e}^{A_{i} t}\right\|\left\|x^{0}\right\|$ is bounded for all $t \geq 0$ and any bounded $\left\|x^{0}\right\|$. This shows that the equilibrium $x^{e}=0$ is stable. Moreover, $\lim _{t \rightarrow \infty} x\left(t, x^{0}\right) \equiv \lim _{t \rightarrow \infty} \mathrm{e}^{A_{i} t} x^{0}=0$ for all $x^{0}$. As a result, the equilibrium is asymptotically stable.

(a) $\Rightarrow(\mathrm{c})$. Since the system is asymptotically stable and thus exponentially stable, by [10], Theorem 5.2, there exists a B-differentiable Lyapunov function $V(z) \equiv \int_{0}^{T} x(t, z)^{T} x(t, z) \mathrm{d} t$ for some $T>0$ such that its directional derivative along the right-hand side of the CLS, denoted by $f(x)$, satisfies $V^{\prime}(z ; f(z)) \leq-c\|z\|^{2}, \forall z$ for some scalar $c>0$. Let $Q_{i}:=\int_{0}^{T}\left(\mathrm{e}^{A_{i} t}\right)^{T} \mathrm{e}^{A_{i} t} \mathrm{~d} t$, which is symmetric and positive definite since $A_{i}$ is Hurwitz. The non-switching property implies $V(z)=z^{T} Q_{i} z, \forall z \in \mathcal{X}_{i}$. Moreover, for any $z \in \mathcal{X}_{i}$, we have $z+\tau A_{i} z \in \mathcal{X}_{i}$ 
for all $\tau \geq 0$ sufficient small. Therefore, for all $z \in \mathcal{X}_{i}$,

$$
\begin{aligned}
V^{\prime}(z ; f(z)) & =\lim _{\tau \downarrow 0} \frac{V(z+\tau f(z))-V(z)}{\tau}=\lim _{\tau \downarrow 0} \frac{\left(z+\tau A_{i} z\right)^{T} Q_{i}\left(z+\tau A_{i} z\right)-z^{T} Q_{i} z}{\tau} \\
& =z^{T}\left(A_{i}^{T} Q_{i}+Q_{i} A_{i}\right) z \leq-c\|z\|^{2} .
\end{aligned}
$$

Therefore $-\left(Q_{i} A_{i}+A_{i}^{T} Q_{i}\right)$ is strictly copositive on $\mathcal{X}_{i}$. Finally, the continuity of $V(z)$ follows from the solution uniqueness and the nonswitching property, i.e., $\mathrm{e}^{A_{i} t} x=\mathrm{e}^{A_{j} t} x, \forall t \geq 0$ for $x \in \mathcal{X}_{i} \cap \mathcal{X}_{j}$.

(c) $\Rightarrow$ (a). Define $V(z):=z^{T} Q_{i} z, \forall z \in \mathcal{X}_{i}$, where $Q_{i}$ 's satisfy the conditions in (c). Since each $Q_{i}$ is symmetric and positive definite, we have $c_{1}\|z\|^{2} \leq V(z) \leq c_{2}\|z\|^{2}, \forall z \in \mathbb{R}^{n}$, where $c_{1}:=\min _{i} \lambda_{\min }\left(Q_{i}\right)$ and $c_{2}:=\max _{i} \lambda_{\max }\left(Q_{i}\right)$ are positive scalars. We next show that $V(z)$ is Lipschitz continuous in the closed unit ball $\mathcal{B}$ of the origin and globally directionally differentiable. Let $L:=\max _{i, j}\left\{\left\|Q_{i}\right\|+\left\|Q_{i}-Q_{j}\right\|\right\}$. For any $z^{1} \in \mathcal{X}_{i} \cap \mathcal{B}$ and $z^{2} \in \mathcal{X}_{j} \cap \mathcal{B}$, we have

$$
\left|V\left(z^{1}\right)-V\left(z^{2}\right)\right| \leq\left|\left(z^{1}\right)^{T} Q_{i} z^{1}-\left(z^{2}\right)^{T} Q_{i} z^{1}+\left(z^{1}\right)^{T} Q_{i} z^{2}-\left(z^{2}\right)^{T} Q_{j} z^{2}\right| \leq L\left\|z^{1}-z^{2}\right\|
$$

Hence, $V$ is Lipschitz continuous in $\mathcal{B}$. Moreover, for any $z, d \in \mathbb{R}^{n}$, we deduce, via [11], Lemma 3.4, that $z+\tau d \in \mathcal{X}_{i}$ for all $\tau \geq 0$ sufficiently small for some $i \in \mathcal{I}(z)$. Hence, $V(z+\tau d)=(z+\tau d)^{T} Q_{i}(z+\tau d)$ for all $\tau \geq 0$ sufficiently small. This immediately yields the existence of the directional derivative $V^{\prime}(z ; d)$ and shows that $V^{\prime}(z ; d)=2 z^{T} Q_{i} d$. Finally, we show that $V^{\prime}(z ; f(z)) \leq-c_{3}\|z\|^{2}, \forall z \in \mathbb{R}^{n}$ for some scalar $c_{3}>0$. Since $-\left(Q_{i} A_{i}+A_{i}^{T} Q_{i}\right)$ is strictly copositive on $\mathcal{X}_{i}$, a positive scalar $\mu_{i}$ exists such that $-z^{T}\left(Q_{i} A_{i}+A_{i}^{T} Q_{i}\right) z \geq$ $\mu_{i}\|z\|^{2}, \forall z \in \mathcal{X}_{i}$. Letting $c_{3}:=\min _{i} \mu_{i}$ and following the similar line discussed before for the directional derivative of $V(z)$, we see that for any $z \in \mathcal{X}_{i}, V^{\prime}(z ; f(z))=z^{T}\left(Q_{i} A_{i}+A_{i}^{T} Q_{i}\right) z \leq-\mu_{i}\|z\|^{2} \leq-c_{3}\|z\|^{2}$. This result, along with [10], Theorem 5.2 and positive homogeneity of the CLSs, leads to exponential stability at the origin.

Theorem 6.6 shows that stability of switch-free CLSs can be easily characterized. The extent to which such a characterization holds for CLSs of other kinds is not known currently; see the recent paper [25] that may provide a negative answer for the existence of piecewise quadratic Lyapunov functions for exponential/asymptotic stability of the planar bimodal CLS with infinite switchings. Finally, it is worth pointing out that the implication (c) $\Rightarrow$ (a) in the theorem holds for general CLSs, even without the switch-free property.

\section{A. Appendix}

Proof of Theorem 6.3. Let $L$ be the global Lipschitz constant for the right-hand side of the CLS (2.1). For a given $\delta>0$ and a scalar $0<c<1$, there is a scalar $T_{\delta, c}>0$ such that

$$
\left\|x^{0}\right\| \leq \delta \Rightarrow\left\|x\left(t, x^{0}\right)\right\| \leq c \delta, \forall t \geq T_{\delta, c}
$$

Moreover, for any $x^{0} \in \mathbb{R}^{n},\left\|x\left(t, x^{0}\right)\right\| \leq \mathrm{e}^{L t}\left\|x^{0}\right\|$ for all $t \geq 0$. Let $\kappa:=\mathrm{e}^{L T_{\delta, c}}$. We have $\left\|x\left(t, x^{0}\right)\right\| \leq$ $\kappa\left\|x^{0}\right\|, \forall t \in\left[0, T_{\delta, c}\right], \forall x^{0} \in \mathcal{B}_{\delta}$. Let $x\left(t, x^{0}\right)$ be the state trajectory with initial state $0 \neq x^{0} \in \mathcal{B}_{\delta}$. The linear property and positive homogeneity of the CLS admits that $\frac{\delta}{\left\|x^{0}\right\|} x\left(t, x^{0}\right)$ is also the state trajectory with initial state $\frac{\delta}{\left\|x^{0}\right\|} x^{0}$ whose norm is $\delta$. Thus by the implication (A.1), we have $\left\|\frac{\delta}{\left\|x^{0}\right\|} x\left(T_{\delta, c}, x^{0}\right)\right\| \leq c \delta$ or equivalently $\left\|x\left(T_{\delta, c}, x^{0}\right)\right\| \leq c\left\|x^{0}\right\| \leq \delta$. Now consider $x\left(t, x^{0}\right)$ on $\left[T_{\delta, c}, 2 T_{\delta, c}\right]$. Since $x\left(t, x^{0}\right)=x\left(s, x\left(T_{\delta, c}, x^{0}\right)\right)$ where $s \in\left[0, T_{\delta, c}\right]$, we have $\left\|x\left(t, x^{0}\right)\right\|=\left\|x\left(s, x\left(T_{\delta, c}, x^{0}\right)\right)\right\| \leq \kappa\left\|x\left(T_{\delta, c}, x^{0}\right)\right\| \leq \kappa c\left\|x^{0}\right\|, \forall t \in\left[T_{\delta, c}, 2 T_{\delta, c}\right]$ and $\left\|x\left(2 T_{\delta, c}, x^{0}\right)\right\|=\left\|x\left(T_{\delta, c}, x\left(T_{\delta, c}, x^{0}\right)\right)\right\| \leq c\left\|x\left(T_{\delta, c}, x^{0}\right)\right\| \leq c^{2}\left\|x^{0}\right\|$. An induction argument can be used to show that $\left\|x\left(t, x^{0}\right)\right\| \leq \kappa c^{n}\left\|x^{0}\right\|$ for all $t \in\left[n T_{\delta, c},(n+1) T_{\delta, c}\right]$ and $\left\|x\left(n T_{\delta, c}\right)\right\| \leq c^{n}\left\|x^{0}\right\|$, for all $n=0,1,2, \ldots$ 
Note that $n \leq t / T_{\delta, c} \leq n+1$. Hence, we have $\left\|x\left(t, x^{0}\right)\right\| \leq \kappa\left\|x^{0}\right\| c^{t / T_{\delta, c}}$ for all $t \geq 0$. Moreover, since $c=\mathrm{e}^{\ln c}$ and $\ln c<0$, we have $\left\|x\left(t, x^{0}\right)\right\| \leq \kappa\left\|x^{0}\right\| \mathrm{e}^{-\gamma t}, \forall t \geq 0$, where $\gamma:=-\ln c / T_{\delta, c}>0$. Since $x^{0}$ and $\delta>0$ are arbitrary, this shows (global) exponential stability.

Acknowledgements. The authors are grateful to Dr. Muruhan Rathinam of University of Maryland Baltimore County (UMBC) for helpful discussions on Lemma 4.5. They thank Dr. Seetharam Gowda of UMBC for bringing their attention to the characterization in Remark 5.3. They also thank the referee and the associate editor for their constructive comments and pointing out the results in [25].

\section{REFERENCES}

[1] A. Arapostathis and M.E. Broucke, Stability and controllability of planar conewise linear systems. Systems Control Lett. 56 (2007) $150-158$.

[2] V.I. Arnold, Mathematical Methods of Classical Mechanics. Second Edition, Springer-Verlag, New York (1989).

[3] S. Basu, R. Pollack and M.-F. Roy, Algorithms in Real Algebraic Geometry. Springer-Verlag (2003).

[4] A. Berman, M. Neumann and R.J. Stern, Nonnegative Matrices in Dynamical Systems. John Wiley \& Sons, New York (1989).

[5] S.P. Bhat and D.S. Bernstein, Lyapunov analysis of semistability, in Proceedings of 1999 American Control Conference, San Diego (1999) 1608-1612.

[6] J. Bochnak, M. Coste and M.-F. Roy, Real Algebraic Geometry. Springer (1998).

[7] N.K. Bose, Applied Multidimensional Systems Theory. Van Nostrand Reinhold (1982).

[8] B. Brogliato, Some perspectives on analysis and control of complementarity systems. IEEE Trans. Automat. Contr. 48 (2003) 918-935.

[9] M.K. Çamlibel, W.P.M.H. Heemels and J.M. Schumacher, On linear passive complementarity systems. European J. Control 8 (2002) 220-237.

[10] M.K. Çamlıbel, J.S. Pang and J. Shen, Lyapunov stability of complementarity and extended systems. SIAM J. Optim. 17 (2006) 1056-1101.

[11] M.K. Çamlibel, J.S. Pang and J. Shen, Conewise linear systems: non-Zenoness and observability. SIAM J. Control Optim. 45 (2006) 1769-1800.

[12] M.K. Çamlibel, W.P.M.H. Heemels and J.M. Schumacher, Algebraic necessary and sufficient conditions for the controllability of conewise linear systems. IEEE Trans. Automat. Contr. 53 (2008) 762-774.

[13] C.T. Chen, Linear System Theory and Design. Oxford University Press, Oxford (1984).

[14] R.W. Cottle, J.S. Pang and R.E. Stone, The Linear Complementarity Problem. Academic Press Inc., Cambridge (1992).

[15] F. Facchinei and J.S. Pang, Finite-Dimensional Variational Inequalities and Complementarity Problems. Springer-Verlag, New York (2003).

[16] D. Goeleven and B. Brogliato, Stability and instability matrices for linear evoluation variational inequalities. IEEE Trans. Automat. Contr. 49 (2004) 483-490.

[17] L. Han and J.S. Pang, Non-Zenoness of a class of differential quasi-variational inequalities. Math. Program. Ser. A 121 (2009) 171-199.

[18] W.P.M.H. Heemels, J.M. Schumacher and S. Weiland, Linear complementarity systems. SIAM J. Appl. Math. 60 (2000) 1234-1269.

[19] J.P. Hespanha, Uniform stability of switched linear systems: extension of LaSalle's invariance principle. IEEE Trans. Automat. Contr. 49 (2004) 470-482.

[20] J.P. Hespanha, D. Liberzon, D. Angeli and E.D. Sontag, Nonlinear norm-observability notions and stability of switched systems. IEEE Trans. Automat. Contr. 50 (2005) 154-168.

[21] H. Khalil, Nonlinear Systems. Second Edition, Prentice Hall (1996).

[22] J. Kurzweil, On the inversion of Lyapunov's second theorem on stability of motion. American Math. Soc. Translation 24 (1963) 19-77.

[23] D. Liberzon, J.P. Hespanha and A.S. Morse, Stability of switched systems: a Lie-algebraic condition. Systems Control Lett. 37 (1999) 117-122.

[24] J. Lygeros, K.H. Johansson, S.N. Simic, J. Zhang and S. Sastry, Dynamic properties of hybrid automata. IEEE Trans. Automat. Contr. 48 (2003) 2-17. 
[25] P. Mason, U. Boscain and Y. Chitour, Common polynomial Lyapunov functions for linear switched systems. SIAM J. Control Optim. 45 (2006) 226-245.

[26] A.P. Molchanove and Y.S. Pyatnitskiy, Criteria of asymptotic stability of differential and difference inclusions encountered in control theory. Systems Control Lett. 13 (1989) 59-64.

[27] M. Pachter and D.H. Jacobson, Observability with a conic observation set. IEEE Trans. Automat. Contr. 24 (1979) $632-633$.

[28] J.S. Pang and J. Shen, Strongly regular differential variational systems. IEEE Trans. Automat. Contr. 52 (2007) $242-255$.

[29] J.S. Pang and D. Stewart, Differential variational inequalities. Math. Program. Ser. A 113 (2008) 345-424.

[30] P.A. Parrilo, Semidefinite programming relaxations for semialgebraic problems. Math. Program. Ser. B 96 (2003) $293-320$.

[31] S. Scholtes, Introduction to Piecewise Differentiable Equations. Habilitation thesis, Institut für Statistik und Mathematische Wirtschaftstheorie, Universität Karlsruhe, Germany (1994).

[32] J.M. Schumacher, Complementarity systems in optimization. Math. Program. Ser. B 101 (2004) 263-295.

[33] J. Shen and J.S. Pang, Linear complementarity systems: Zeno states. SIAM J. Control Optim. 44 (2005) 1040-1066.

[34] J. Shen and J.S. Pang, Linear complementarity systems with singleton properties: non-Zenoness, in Proceedings of 2007 American Control Conference, New York (2007) 2769-2774.

[35] J. Shen and J.S. Pang, Semicopositive linear complementarity systems. Internat. J. Robust Nonlinear Control 17 (2007) $1367-1386$.

[36] G.V. Smirnov, Introduction to the Theory of Differential Inclusions, Graduate Studies in Mathematics 41. American Mathematical Society, Providence (2002). 